\title{
A Chandra and ALMA Study of X-ray-irradiated Gas in the Central $\sim 100$ pc of the Circinus Galaxy
}

\author{
Taiki Kawamuro* ${ }^{* 1, \dagger}$, Takuma Izumi $\mathbf{i}^{1, \ddagger}$ and Masatoshi Imanishi ${ }^{1,2}$ \\ ${ }^{1}$ National Astronomical Observatory of Japan, Osawa, Mitaka, Tokyo 181-8588, Japan \\ ${ }^{2}$ Department of Astronomy, School of Science, Graduate University for Advanced Studies \\ (SOKENDAI), Mitaka, Tokyo 181-8588 \\ $\dagger J S P S$ fellow (PD) \\ $\ddagger N A O J$ fellow \\ *E-mail: taiki.kawamuro@nao.ac.jp \\ Received 〈reception date); Accepted 〈acception date〉
}

\begin{abstract}
We report a study of X-ray-irradiated gas in the central $\sim 100 \mathrm{pc}$ of the Circinus galaxy, hosting a Compton-thick active galactic nucleus (AGN), at 10-pc resolution using Chandra and ALMA. Based on $\sim 200 \mathrm{ksec}$ Chandra/ACIS-S data, we created an image of the $\mathrm{Fe} \mathrm{K} \alpha$ line at 6.4 $\mathrm{keV}$, tracing X-ray-irradiated dense gas. The ALMA data in Bands $6(\sim 270 \mathrm{GHz})$ and $7(\sim 350$ $\mathrm{GHz})$ cover five molecular lines: $\mathrm{CO}(J=3-2), \mathrm{HCN}(J=3-2), \mathrm{HCN}(J=4-3), \mathrm{HCO}^{+}(J=3-2)$, and $\mathrm{HCO}^{+}(J=4-3)$. The detailed spatial distribution of dense molecular gas was revealed, and compared to the iron line image. The molecular gas emission appeared faint in regions with bright iron emission. Motivated by this, we quantitatively discuss the possibility that the molecular gas is efficiently dissociated by AGN X-ray irradiation (i.e., creating an X-ray-dominated region). Based on a non-local thermodynamic equilibrium model, we constrained the molecular gas densities and found that they are as low as interpreted by X-ray dissociation. Furthermore, judging from inactive star formation (SF) reported in the literature, we suggest that the $\mathrm{X}$-ray emission has potential to suppress SF, particularly in the proximity of the AGN.
\end{abstract}

Key words: galaxies: active $_{1}-$ galaxies: individual (the Circinus galaxy) $)_{2}-\mathrm{X}$-rays: galaxies $_{3}-$ submm/mm: galaxies 4

\section{INTRODUCTION}

Active galactic nuclei (AGN) liberate the enormous gravitational energy of the mass accreted by supermassive black holes (SMBHs) into their host galaxies. As an extreme case, theoretical works have suggested that AGN are energetic enough to terminate galaxy evolution, or star formation (SF), by blowing out the surrounding gas and heating the halo to prevent cooling flows (e.g., Silk \& Rees 1998; Di Matteo et al. 2005; Croton et al. 2006; Fabian 2012). Then, many observational investigations on how the AGN interacts with the host galaxy have been conducted (e.g., Sturm et al. 2011; Page et al. 2012; Harrison et al. 2012; Tombesi et al. 2015, 2017; Fiore et al. 2017; Woo et al. 2017). Among such studies, spatially resolved spectroscopy has played an important role in mapping and/or quantifying AGN effects such as ionized gas and outflows (e.g., Feruglio et al. 2010; Liu et al. 2013a, 2013b; Cicone et al. 2014; Harrison et al. 2014; Bae \& Woo 2016; Bae et al. 2017; Kawamuro et al. 2017; Oosterloo et al. 2017). Furthermore, the spatial associations with the SF have been examined, and the results have showed that

(C) 2018. Astronomical Society of Japan. 
AGN may suppress and/or promote SF (e.g., Cano-Díaz et al. 2012; Cresci et al. 2015a, 2015b; Feruglio et al. 2015; Maiolino et al. 2017).

AGN are usually luminous in the X-ray regime, and thus an unavoidable AGN effect is X-ray irradiation, which can ionize and heat the surrounding gas. This region, referred to as the X-ray-dominated region (XDR; e.g., Maloney 1999), is more expansive than the so-called photodissociation region by ultraviolet (UV) photons due to the high column-penetrating power. Thus far, great efforts have been made to understand the physical (i.e., size, density, and temperature; Proga et al. 2014) and chemical conditions (e.g., Lepp \& Dalgarno 1996; Maloney et al. 1996, 1999; Usero et al. 2004; Meijerink \& Spaans 2005; Meijerink et al. 2007; Viti et al. 2014) in the XDR. Among processes suggested, an interesting one is that photoelectrons produced by X-rays dissociate molecules. This may consequently suppress SF activity, given the positive correlation between the molecular gas and $\mathrm{SF}$ rate $\mathrm{SFR}$ ) surface densities (e.g., Kennicutt et al. 2007; Bigiel et al. 2008), suggesting a causal link between the chemical state of interstellar matter and the ability to form stars.

Mapping the $\mathrm{Fe} \mathrm{K} \alpha$ fluorescent line at $6.4 \mathrm{keV}$ is a simple way to identify X-ray-irradiated gas. This line is produced through ionization by X-rays with energies above $7.1 \mathrm{keV}$, corresponding to the K-edge of neutral iron. A medium becomes optically thick for the $7.1 \mathrm{keV}$ photon around $\log N_{\mathrm{H}} / \mathrm{cm}^{-2} \sim 23.9$, given the low crosssection and the abundance $\left(\sigma_{\mathrm{Fe}} \approx 3.5 \times 10^{-20} \mathrm{~cm}^{2}\right.$ and $A_{\mathrm{Fe}} \approx 3.3 \times 10^{-5}$; Morrison \& McCammon 1983). As such, the $\mathrm{Fe} \mathrm{K} \alpha$ line has some advantages to investigate regions of X-ray-irradiated dense gas, such as the galaxy center. First, the low cross-section is convenient for tracing the entire region of dense gas; otherwise, only the surface could be probed. Second, the resultant Fe K $\alpha$ photons still retain good penetrating power, and therefore AGN X-ray-affected regions can be unveiled with a small bias against the absorption. Finally, X-rays at energies above the Fe K edge are little contaminated by optically thin thermal emission from the SF (e.g., LaMassa et al. 2012; Kawamuro et al. 2016). So far, Chandra, which achieves the highest angular resolution in the X-ray band, has played a leading role in the investigation of morphological X-ray properties, and has successfully unveiled the extended Fe $\mathrm{K} \alpha$ emission of some nearby AGN host galaxies (e.g., NGC 4945, NGC 1068, the Circinus galaxy, and ESO 428-G014; Young et al. 2001; Marinucci et al. 2012, 2013, 2017; Fabbiano et al. 2017).

In this study, we investigated the physical and chemical conditions of X-ray-irradiated gas at high spatial resolutions, to confirm whether X-rays dissociate molecular gas. For this purpose, we studied the central $\sim 100$ pc of the Circinus galaxy (hereafter, Circinus), which hosts a Compton-thick AGN (Matt et al. 1999), using Chandra and ALMA. Both observatories can achieve spatial resolutions down to sub-arcsec. The redshift, systematic velocity, and distance of the galaxy are respectively $z=0.001448$, $v=434 \mathrm{~km} \mathrm{~s}^{-1}$ (Koribalski et al. 2004), and $4.2 \mathrm{Mpc}$ (e.g., Karachentsev et al. 2013), where 1 arcsec corresponds to 20 pc. The high spatial resolution, achievable given the proximity, is crucial in discussion of the AGN effect, with the SF effect being discriminated as much as possible (e.g., Izumi et al. 2016). In addition, suppression of the transmitted emission by the Compton-thick material makes it easy to detect the faint extended X-ray emission. Thus, Circinus is one of the best laboratories for studying X-ray-irradiated gas. The AGN center, or the SMBH position, is RA, Decl. $(\mathrm{J} 2000)=14 \mathrm{~h} 13 \mathrm{~m} 09.953 \mathrm{~s},-65 \mathrm{~d} 20 \mathrm{~m} 21.047 \mathrm{~s}$, determined by the $22-\mathrm{GHz} \mathrm{H}_{2} \mathrm{O}$ maser observation (Greenhill et al. 2003).

The remainder of this paper is organized as follows. Section 2 briefly summarizes what have been observed in Circinus. Section 3 presents an overview of Chandra and ALMA data. Chandra X-ray images and spectra are analyzed in Section 4, and three subregions of interest are defined. Molecular gas properties in the subregions are derived using ALMA data in Section 5. Our discussion and summary are given in Sections 6 and 7, respectively. Unless otherwise noted, errors are quoted at the $1 \sigma$ confidence level for a single parameter of interest.

\section{CIRCINUS}

Circinus has been observed at various wavelengths, and good quality and/or spatially well-resolved data, obtained thanks to the proximity, indicate the presence of an AGN in the center. Dust lanes with an $\sim 100$ pc scale are located in the galaxy plane and contribute to the nuclear obscuration (Wilson et al. 2000), but optical and near-infrared (IR) spectroscopies have captured AGN signatures such as strong coronal emission lines (Oliva et al. 1994; Moorwood et al. 1996). One-side ionization cones in the northwest direction, likely originating from the AGN, were also imaged at $\mathrm{H} \alpha$ and [O III] (Marconi et al. 1994; Wilson et al. 2000). Further, a near-IR [Si VII] line observation unveiled a cone in the opposite direction (Prieto et al. 2005). As a signature more closely related to the AGN, the broad $\mathrm{H} \alpha$ emission line was observed in polarized light (Oliva et al. 1998). $\mathrm{X}$-ray data have also given beneficial information. Soft Xray observations detected flat continuum together with a strong iron line, indicating Compton scattering and fluorescent emission from gas illuminated by an X-ray source 
Table 1. Chandra Data List

\begin{tabular}{cccc}
\hline \hline ObsID & Obs. date (UT) & Grating & $\begin{array}{c}\text { Exp. } \\
(\mathrm{ksec})\end{array}$ \\
$(1)$ & $(2)$ & $(3)$ & $(4)$ \\
\hline 12823 & $2010 / 12 / 17$ & NO & 147 \\
12824 & $2010 / 12 / 24$ & NO & 38 \\
& & (Total & $185)$ \\
\hline 62877 & $2000 / 06 / 16$ & YES & 48 \\
4770 & $2004 / 06 / 02$ & YES & 48 \\
4771 & $2004 / 11 / 28$ & YES & 52 \\
& & (Total & $148)$ \\
\hline
\end{tabular}

(1) Observation ID. (2) Observation start date. (3) Check on the grating observation. (4) Exposure after data reduction.

(Matt et al. 1996; Smith \& Wilson 2001). Then, hard $\mathrm{X}$-ray observations confirmed the central engine heavily obscured by a large column density $\left(\log N_{\mathrm{H}} / \mathrm{cm}^{-2} \sim 24-\right.$ 25; Matt et al. 1999; Soldi et al. 2005; Yang et al. 2009; Arévalo et al. 2014)

The SF is also a topic extensively discussed so far. Marconi et al. (1994) reported $\sim 200$ pc scale star forming rings (see also Elmouttie et al. 1998b), and molecular gas distributions at similar spatial scales were also confirmed at various emission lines (e.g., Elmouttie et al. 1998c; Curran et al. 1998, 1999; Izumi et al. 2018). The SFR in the rings was estimated to be a few $M_{\text {sun }} \mathrm{yr}^{-1}$, but it decreases at scales $<100 \mathrm{pc}$ to be $\sim 0.01-0.1 M_{\text {sun }} \mathrm{yr}^{-1}$ (Maiolino et al. 2000; Esquej et al. 2014).

\section{OBSERVATION DATA}

\subsection{Chandra Observations}

Chandra observations towards Circinus were made 10 times with the Chandra/Advanced CCD Imaging Spectrometer in the $1 \times 6$ array CCD configuration (ACISS; Garmire et al. 2003). Their focal points, where the highest spatial resolution can be achieved, were closely set to the nucleus $(\approx 0.3$ arcmin $)$. Out of the ten, the High-Energy Transmission Grating (HETG; Canizares et al. 2005), consisting of two sets of gratings: the HighEnergy Grating (HEG) and the Medium-Energy Grating, was used four times.

As summarized in Table 1, we utilized two data obtained without the HETG grating (ObsID = 12823 and 12824) to discuss spatial distributions of X-ray emission. The remaining four among the six non-grating data were ignored, because of small contributions to the total exposure ( $\sim 200$ ksec). Marinucci et al. (2013) already reported
X-ray spectral and image analyses using the same ObsID $=12823$ and 12824 data, but we made additional efforts to discriminate between the nuclear and extended emission (Sections 4.3 and 4.4).

Moreover, among the four grating data of all the ten, we used three data (ObsID $=4770,4771$, and 62877), each obtained with a larger exposure of $\approx 60 \mathrm{ksec}$ than the remaining one $(\approx 7 \mathrm{ksec})$. A spectrum of the nuclear point source was constrained from them, and subsequently was used to determine how largely the nuclear X-ray emission contaminates spectra extracted from outer regions. That was also utilized to estimate fractions of the pile-up, in which more than one photon is counted as one photon within a single readout. Particularly around the nucleus, the pileup effect cannot be ignored. Although the 0th order of the grating data is equivalent to the imaging, we did not take account of it for our main imaging analysis because of possible calibration uncertainties (Arévalo et al. 2014). In Appendix A, we however present a result that includes the 0th order data and find it consistent with our main result. Note that in this supplemental analysis, we additionally take account of usable data obtained with an aim to study a nearby object of SN 1996cr, 20 arcsec away from Circinus. Most of them were taken in the grating mode.

\subsection{ALMA Observations}

We analyzed Band $6 \quad(\sim 270 \quad \mathrm{GHz})$ and $7 \quad(\sim 350$ $\mathrm{GHz}$ ) ALMA datasets obtained through two programs \#2015.1.01286.S (PI: F. Costagliola) and \#2016.1.01613.S (PI: T. Izumi), as summarized in Table 2. In the former program, two observations with short on-source exposures ( $\sim$ a few minutes) were executed. They covered five molecular lines: $\operatorname{CO}(J=3-2)$ at the rest-frame frequency of $\nu_{\text {rest }}$ $=345.796 \mathrm{GHz}, \mathrm{HCO}^{+}(J=3-2)$ at $\nu_{\text {rest }}=267.558 \mathrm{GHz}$, $\mathrm{HCO}^{+}(J=4-3)$ at $\nu_{\text {rest }}=356.734 \mathrm{GHz}, \mathrm{HCN}(J=3-2)$ at $\nu_{\text {rest }}=265.886 \mathrm{GHz}$, and $\operatorname{HCN}(J=4-3)$ at $\nu_{\text {rest }}=354.505$ $\mathrm{GHz}$. We used this program to estimate their line ratios, and finally to discuss properties of the molecular gas (Sections 5.1.2, 6.2, and 6.3). This allowed us to ignore the systematic uncertainty in the absolute flux as much as possible that can be caused between different observations. On the other hand, the latter program yielded much better-quality data for $\mathrm{CO}(J=3-2)$ and $\mathrm{HCO}^{+}(J=4-3)$ using a long exposure $(\sim 2 \mathrm{~h})$. In this paper, we analyze only the $\mathrm{HCO}^{+}(J=4-3)$ data to reveal the dense molecular gas distribution in great detail by exploiting the high signal to noise ratio (SNR). An analysis of the $\mathrm{CO}(J=3-2)$ line data was already reported by Izumi et al. (2018).

$\mathrm{HCN}(J=4-3), \mathrm{HCO}^{+}(J=4-3)$, and $\mathrm{CO}(J=3-2)$ lines 
Table 2. ALMA Data List

\begin{tabular}{lllll}
\hline \hline Tag & Program Info. & Obs. date (UT) & $\begin{array}{l}\text { Exp. } \\
(\min )\end{array}$ & Molecules \\
& & $(3)$ & $(4)$ & $(5)$ \\
\hline (a) & \#2015.1.01286.S (PI: F. Costagliola) & $2015 / 12 / 31$ & 3 & $\mathrm{HCO}^{+}(J=4-3), \mathrm{HCN}(J=4-3), \mathrm{CO}(J=3-2)$ \\
(a) & $\# 2015.1 .01286 . \mathrm{S}$ (PI: F. Costagliola) & $2015 / 12 / 31$ & 5 & $\mathrm{HCO}^{+}(J=3-2), \mathrm{HCN}(J=3-2)$ \\
(b) & $\# 2016.1 .01613 . \mathrm{S}$ (PI: T. Izumi) & $2016 / 11 / 24$ & 125 & $\mathrm{HCO}^{+}(J=4-3)$ \\
\hline Notes.
\end{tabular}

(1) (a) The dataset was used mainly to constrain and discuss the physical and chemical properties of the molecular gas (Sections 5.1.2, 6.2, and 6.3). (b) The dataset was used to reveal the dense molecular gas distribution to be compared to the Fe K $\alpha$ line image (Section 6.1). (2) Project identification and principal investigator. (3) Observation start date. (4) Total on-source exposure time. (5) Molecular emission line(s) taken from each data.

were observed simultaneously in Band 7 on 2015 December 31, when 38 antennas were operated. The maximum recoverable scale (MRS), the largest angular scale structure that can be recovered from observations, was 7 arcsec. This is large enough to discuss the central $\approx 100 \mathrm{pc}$, or 5 arcsec, scale structure. Out of four spectral windows, three were used to observe the lines. Each window had a total bandwidth of $1.875 \mathrm{GHz}$ and 240 channels. The raw spectral resolution was $7.8 \mathrm{MHz}\left(6.8 \mathrm{~km} \mathrm{~s}^{-1}\right)$, but we binned three spectral elements to achieve a resolution of $23 \mathrm{MHz}(20 \mathrm{~km}$ $\mathrm{s}^{-1}$ ), as explained in Section 5.1. The total on-source time was $\approx 0.05 \mathrm{~h}$ (3 $\mathrm{min})$. Standard flux, bandpass, and phase calibrations were conducted based on the observations of Titan, J1427-4206, and J1424-6807, respectively.

Band 6 simultaneous observations of $\operatorname{HCN}(J=3-2)$ and $\mathrm{HCO}^{+}(J=3-2)$ lines were made on 2015 December 31 using 36 antennas. A larger MRS of 9 arcsec was achieved because of the lower frequencies. Two of three spectral windows were used for the line detection in the same total bandwidth and number of channels as above. The objects for the calibrations were also the same. The total on-source time was $\approx 0.09 \mathrm{~h}(5 \mathrm{~min})$. As in the case above, a resolution of $16 \mathrm{MHz}\left(18 \mathrm{~km} \mathrm{~s}^{-1}\right)$ was adopted by binning two spectral elements at the raw resolution of $7.8 \mathrm{MHz}(8.9 \mathrm{~km}$ $\left.\mathrm{s}^{-1}\right)$.

We mapped the $\mathrm{HCO}^{+}(J=4-3)$ line using Band 7 data acquired on 2016 November 24 and 26, and 2017 May 05 using 42, 42, and 47 antennas, respectively. The MRS was the same as that for the above $\mathrm{HCO}^{+}(J=4-3)$ line data (i.e., 7 arcsec). Four spectral windows were employed in the observations, each with a total bandwidth of $1.875 \mathrm{GHz}$ and 480 channels. Although two of the spectral windows can be used to detect the $\mathrm{HCO}^{+}(J=4-3)$ line, we used only one that covered a broader frequency range around the $\mathrm{HCO}^{+}(J=4-3)$ line. The total on-source time was $\approx$ $2.1 \mathrm{~h}$ (125 min). The calibration sources for flux, bandpass, and phase were J1427-4206 or J1617-5848, J14274206, and J1424-6807, respectively. Because it is possible

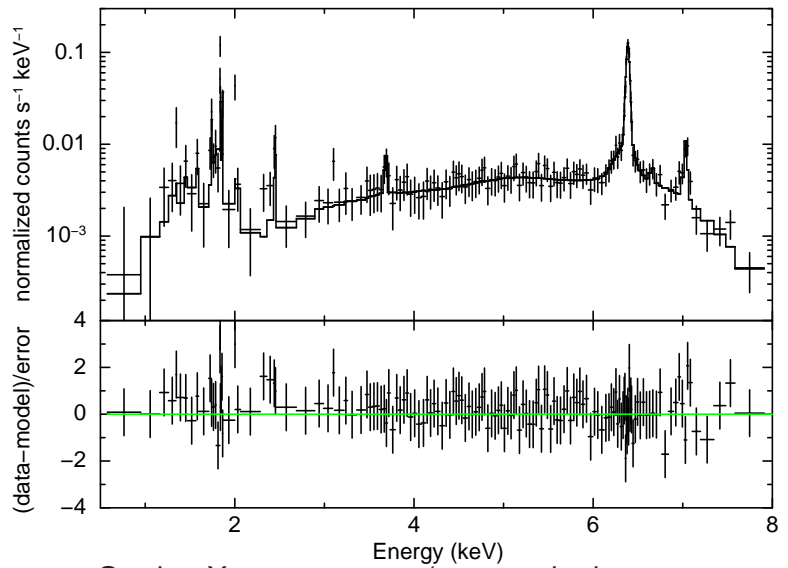

Fig. 1. Grating X-ray spectrum (crosses in the upper panel), folded by the response function. The best-fit model is shown by a solid line. For clarity, all three spectra used for the fit were combined. The lower panel plots the residuals.

to detect broad but faint components using a high SNR, we adopted a finer resolution of $12 \mathrm{MHz}\left(9.8 \mathrm{~km} \mathrm{~s}^{-1}\right)$, which was achieved by binning three spectral elements at the raw resolution of $3.9 \mathrm{MHz}\left(3.3 \mathrm{~km} \mathrm{~s}^{-1}\right)$.

\section{CHANDRA DATA ANALYSES}

\subsection{Reduction and Reprocessing of Chandra Data}

The Chandra data were reduced using the Chandra Interactive Analyses of Observations (CIAO) 4.9 and the Chandra Calibration Data Base 4.7.6, provided by the Chandra team. Following standard procedures, we reprocessed all raw data using the chandra_repro script. Furthermore, we examined the data with deflare to remove background flaring periods when the rates deviated from the mean by more than $3 \sigma$.

We checked the absolute astrometry of the Chandra image based on the SMBH position that was determined by the $\mathrm{H}_{2} \mathrm{O}$ maser observation. We representatively examined ObsID 12823 because of the longest exposure. The 
Table 3. Positional Subregion Information

\begin{tabular}{ccccc}
\hline \hline Region & R.A. (J2000) & Decl. (J2000) & $\begin{array}{c}\text { Sep. angle } \\
(\operatorname{arcsec})\end{array}$ & $\begin{array}{c}\text { Dist. } \\
(\mathrm{pc})\end{array}$ \\
$(1)$ & $(2)$ & $(3)$ & $(4)$ & $(5)$ \\
\hline Nucleus & $14 \mathrm{~h} 13 \mathrm{~m} 09.953 \mathrm{~s}$ & $-65 \mathrm{~d} 20 \mathrm{~m} 21.047 \mathrm{~s}$ & $\ldots$ & $\ldots$ \\
CNR-E & $14 \mathrm{~h} 13 \mathrm{~m} 10.402 \mathrm{~s}$ & $-65 \mathrm{~d} 20 \mathrm{~m} 20.205 \mathrm{~s}$ & 2.9 & 62 \\
CNR-SE & $14 \mathrm{~h} 13 \mathrm{~m} 10.342 \mathrm{~s}$ & $-65 \mathrm{~d} 20 \mathrm{~m} 22.461 \mathrm{~s}$ & 2.8 & 60 \\
\hline
\end{tabular}

(1) Name of the subregions defined in Figure 2 (2) Right ascension. (3) Declination. (4) Separation angle to the nucleus, or the SMBH position. (5) Deprojected physical distance (see detail in Section 4.3).
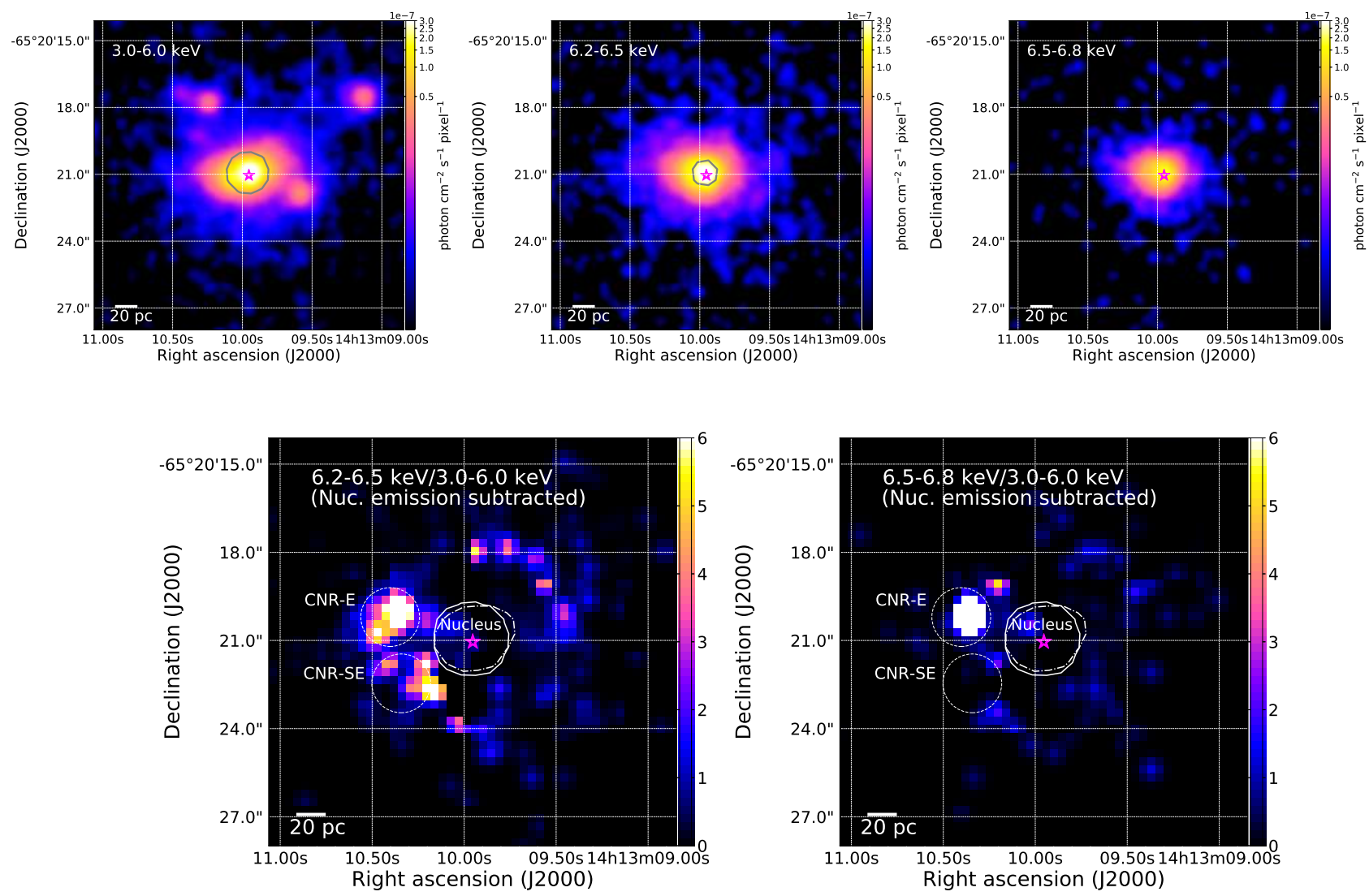

Fig. 2. Top panels show integrated ACIS-S images in different energy bands (from left to right: $3.0-6.0 \mathrm{keV}, 6.2-6.5 \mathrm{keV}$, and $6.5-6.8 \mathrm{keV}$ ) within the central $\approx 14 \times 14 \operatorname{arcsec}^{2}$ region. The size of each pixel is $0.0625 \times 0.0625 \mathrm{arcsec}^{2}$, and smoothing was performed using a Gaussian kernel with FWHM of 0.495 arcsec. The gray solid contours enclose the area with pile-up fractions larger than $5 \%$ for each energy band. Bottom panels show images constructed by dividing two of the three images (from left to right: $6.2-6.5 \mathrm{keV} / 3.0-6.0 \mathrm{keV}$ and $6.5-6.8 \mathrm{keV} / 3.0-6.0 \mathrm{keV}$ ) from which the nuclear point source emission was subtracted. We calculated the ratios with images whose pixel size was $0.25 \times 0.25 \mathrm{arcsec}^{2}$. Smoothing was performed with the same Gaussian kernel as above. Contours corresponding to the pile-up fraction of 5\% determined from the $0.5-8.0 \mathrm{keV}$ simulated data and the observed data via the pileup_map command are shown by solid and dot-dashed lines, respectively. Two dotted circles with a 1-arcsec radius indicate the subregions of CNR-E and CNR-SE. In all of the figures, the magenta star corresponds to the SMBH position, labeled as the Nucleus, the third subregion. 
centroid of the nuclear X-ray emission was likely coincident with the central AGN engine, or the SMBH position. Hence, they were compared to each other. The derived spatial offset was $\approx 0.1$ arcsec, sufficiently small to be ignored when we discuss larger-scale structures $(\gtrsim 0.5$ arcsec). Thus we used it as a basis for the X-ray image.

We corrected the remaining imaging data $(\mathrm{ObsID}=$ 12824) for a systematic offset by spatially matching point sources distributing in both images. We started by making exposure-corrected $0.5-7.0 \mathrm{keV}$ images with fluximage where exposure maps were created with an effective energy of $2.3 \mathrm{keV}$. Also, we looked up the point spread function (PSF) size of each pixel in all images using the mkpsfmap script. Combined with these results, wavdetect was run to create X-ray source lists, containing information on the source angular size and SNR. At last, the adjustment was made by adopting only point-like, bright sources detected with PSF size $<1.5$ arcsec, source angular size $<1.0$ arcsec, and SNR $>7$. We found that the offset was 0.2 arcsec, much smaller than structures of interest. This suggests that even if we choose the ObsID $=12824$ as the basis, it does not affect our discussion.

\subsection{X-ray Emission from Nuclear Point Source}

To properly constrain spatial and spectral properties of extended emission around the bright AGN, we need to take account of contamination by the AGN, possible due to the finite PSF. Here, we simply define the AGN as a point source spatially unresolved with Chandra. For that purpose, we prepared a simulated AGN data.

\subsubsection{Grating Spectra}

We first determined an X-ray spectrum of the AGN. Because the Chandra imaging suffered from the pile-up effect around the nucleus (Section 4.3), we used the grating data, or the HEG data, reprocessed in Section 4.1. We extracted spectra using a 4.8 arcsec full-width in the cross-dispersion direction centered on the AGN. The plus and minus first order spectra were combined to increase the SNR. The spectra were binned so that each energy bin had at least one count. Accordingly adopting the $C$-statistic, appropriate even for low photon counts, we determined the best-fit on XSPEC Version 12.9.1m (Arnaud 1996).

We simultaneously fitted the three spectra in the 0.5 $8.0 \mathrm{keV}$ band with two power-law components and emission lines identified by Sambruna et al. (2001). They conducted a spectral analysis for the ObsID $=62877$ HETG data. Because residuals were left in the fit, we further added several Gaussian components and obtained a good fit with $C$ statistic/degrees of freedom (d.o.f) $=2670 / 3130$. Figure 1 shows the fitting result. The precise measurement of the spectral shape is the main aim of this analysis, and the physical interpretation is beyond the scope of this work.

\subsubsection{MARX Simulation}

Next, we simulated imaging observations of a point source having the $0.5-8.0 \mathrm{keV}$ spectral shape determined in Section 4.2.1. We utilized the MARX Version 5.3.3 (Davis et al. 2012), which performs ray-tracing simulations that take account of the mirror and detector responses. Simulation parameters, such as nominal position, start time, and exposure, were set to those of each of the two observations (ObsID = 12823 and 12824). The source position was set to the X-ray peak found in the $3-6 \mathrm{keV}$ band image. Even if we adopt the SMBH position instead, our conclusion does not change. To reduce statistical fluctuations of the simulated models, we performed 100 MARX simulations for each observation, and took their average. Because the pile-up was ignored in the simulation as default, we also created pile-up affected data according to a standard procedure ${ }^{\mathrm{i}}$. Then, we confirmed good agreement between the simulated and observed data by comparing their central 1 -arcsec spectra. The data without the pileup is used to confirm regions affected by the pileup at different energy bands by taking advantage of the record of the intrinsic count rates (Section 4.3). The other data is used to subtract the nuclear emission from observed images to reveal the extended emission (Section 4.3), and also is taken into consideration in analyzing spectra at the subregions of interest (Section 4.4).

\subsection{Mapping of Iron Emission Lines}

We probe the X-ray-irradiated dense gas mainly through an $\mathrm{Fe} \mathrm{K} \alpha$ emission line map. Other emission from ionized irons is useful for the discussion as well. Thus, it is mapped supplementarily. First, we create X-ray images based on Energy Dependent Sub-pixel Event Repositioning (e.g., Tsunemi et al. 2001; Mori et al. 2001; Li et al. 2003, 2004) ii, which makes it possible to achieve angular resolutions greater than the CCD pixel size of 0.498 arcsec. The algorithm redistributes photons based on the shape of the charge cloud recorded in a $3 \times 3$ pixel island, while considering the energy dependence. At last, all images are merged into one to increase the SNR using merge_obs.

The upper panels of Figure 2 show the $3.0-6.0 \mathrm{keV}$, $6.2-6.5 \mathrm{keV}$, and $6.5-6.8 \mathrm{keV}$ band images, whose expo-

\footnotetext{
${ }^{\text {i }}$ http://cxc.harvard.edu/ciao/threads/marx_sim/

${ }^{\text {ii }}$ More details are available in http://cxc.harvard.edu/ciao/why/acissubpix.html http://cxc.harvard.edu/ciao/guides/acis_subpixel.html
} 

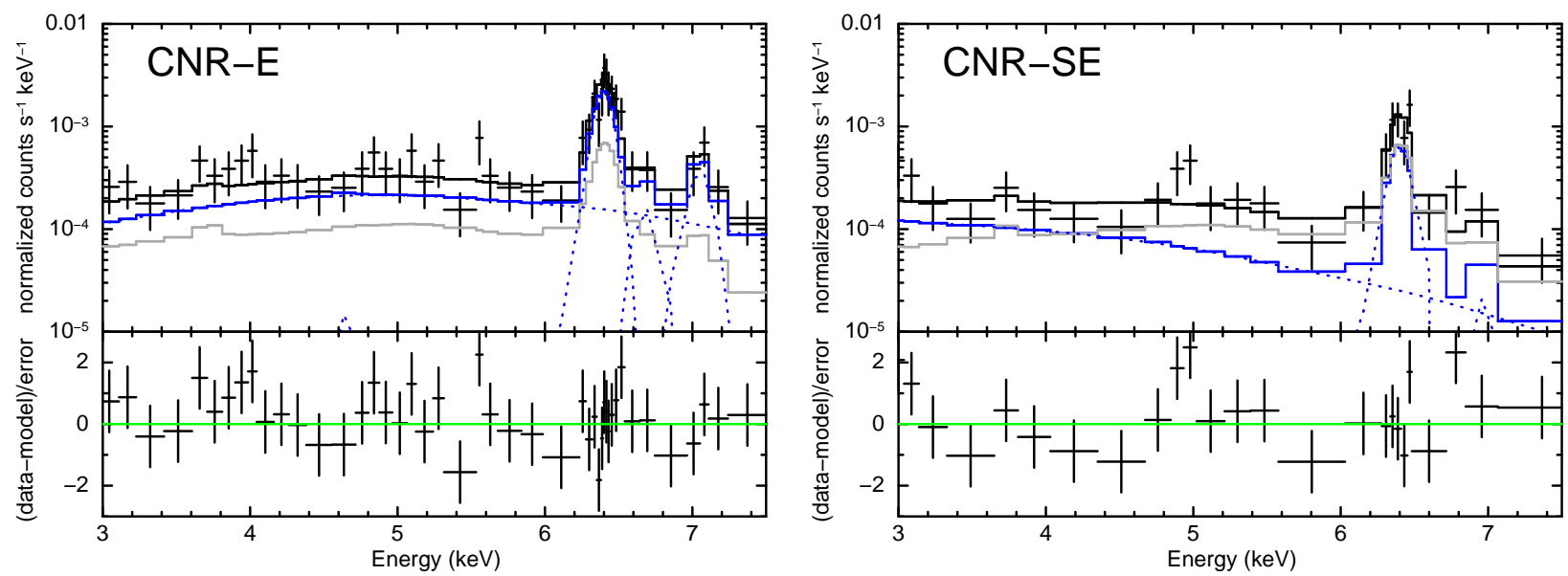

Fig. 3. X-ray spectra (black crosses) extracted from the CNR-E and CNR-SE regions. Each figure represents only a single spectrum taken from the ObsID = 12823 data and rebinned for clarity. The spectra were reproduced by the contaminating $\mathrm{X}$-ray from the nuclear point source (gray) and the extended emission of interest (blue). Lower data represent the residuals.

sures maps are created by representatively adopting their intermediate energies of $4.50 \mathrm{keV}, 6.35 \mathrm{keV}$, and 6.65 $\mathrm{keV}$, respectively. A pixel size of $0.0625 \times 0.0625 \operatorname{arcsec}^{2}$ is adopted, and the images are smoothed via a Gaussian kernel with full width half maximum $($ FWHM $)=0.495$ arcsec. The energy bands are selected to trace the continuum component, the neutral $\mathrm{Fe} \mathrm{K} \alpha$ emission, and the emission from ionized He-like irons (Fe XXV He $\alpha$ ), respectively. Although emission from highly ionized irons (Fe XXVI Ly $\alpha$ ) can be seen at $6.97 \mathrm{keV}$, we ignore it because of the difficulty to isolate the line emission from the neutral Fe $\mathrm{K} \beta$ emission at $7.06 \mathrm{keV}$. The lower panels of Figure 2 show images created by dividing two of the three energy band images. To increase the SNR, pre-divided X-ray images are made by adopting a larger pixel size of $0.25 \times 0.25$ $\operatorname{arcsec}^{2}$. Also, the simulated AGN emission is subtracted to reveal the extended emission. Smoothing is conducted using the Gaussian kernel with FWHM $=0.495$ arcsec. The ratio between the $6.2-6.5 \mathrm{keV}$ and $3.0-6.0 \mathrm{keV}$ images and that between the 6.6-7.0 $\mathrm{keV}$ and $3.0-6.0 \mathrm{keV}$ images correspond to proxies of the $\mathrm{Fe} \mathrm{K} \alpha$ line and ionized iron line equivalent widths, respectively.

Here, we check regions affected by the pile-up. The fraction of piled events to the total detections $\left(f_{\mathrm{p}}\right)$ can be calculated as $f_{\mathrm{p}}=1-\alpha /(\exp (\alpha \Lambda)-1)$ iii. The $\alpha$ parameter is a probability that for each photon event beyond the first, the piled event is identified as a real event, and $\Lambda$ represents the intrinsic counts per detector region per frame time. With $\Lambda$ from the simulated data (Section 4.2.2) and an assumption of $\alpha=1$, corresponding to the most conservative case, the fraction can be estimated for a given

\footnotetext{
iii see also "The Chandra ABC Guide to Pileup" in http://cxc.harvard.edu/ciao/download/doc/pileup_abc.pdf
}

energy band. Figure 2 shows contours surrounding areas with pile-up fractions $>5 \%$. Regarding the images of the ratios, we representatively show contours determined using the $0.5-8.0 \mathrm{keV}$ events. Note that a similar result can be obtained using the CIAO pileup_map task (Figure 2), which approximately calculates the pile-up fraction based on the observed events.

We find that the $\mathrm{Fe} \mathrm{K} \alpha$ emission seems to extend up to $\approx 3 \operatorname{arcsec}(\sim 60 \mathrm{pc})$ to the east and southeast (the lower left panel of Figure 2), consistent with Marinucci et al. (2013). This configuration looks like a conical structure oriented towards the southeast direction, and may be a counterpart of the optical ionization cone found in the northwest direction (Marconi et al. 1994). As denoted in the lower left panel of Figure 2, we define two circum-nuclear regions with moderate Fe K $\alpha$ emission as CNR-E and CNR-SE, which are our main interest in this paper. Also, the central region is labeled as Nucleus. Table 3 lists detailed information on the three subregions. The deprojected distances of the CNR-E and CNR-SE regions are estimated to be $\approx 60 \mathrm{pc}$ from the SMBH. This estimate assumes that the subregions associate with a counterpart of the northwest $\mathrm{H} \alpha$ ionization cone (Elmouttie et al. 1998a), inclined by 70 degrees with respect to our sightline.

\subsection{Spatially Resolved X-ray Spectral Analyses}

Based on X-ray spectral analyses, we confirm that the Fe $\mathrm{K} \alpha$ line is the result of X-ray illumination by the central engine. We extract 3.0-7.5 keV spectra from the two circular regions (CNR-E and CNR-SE) with a 1 -arcsec radius. We exclude the soft X-ray band $(<3 \mathrm{keV})$ because non-AGN emission such as optically thin thermal emission from the 
Table 4. Best-fit Parameters

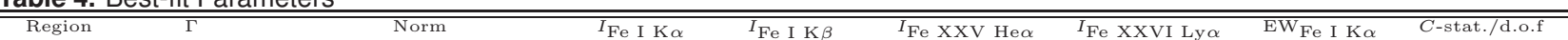

\begin{tabular}{|c|c|c|c|c|c|c|c|c|}
\hline \multirow[b]{3}{*}{ (1) } & \multirow{3}{*}{\multicolumn{2}{|c|}{$\begin{array}{l}\left(10^{-7} \text { photons }\right. \\
\left.\mathrm{keV}^{-1} \mathrm{~cm}^{-2} \mathrm{~s}^{-1}\right) \\
\text { (3) }\end{array}$}} & \multirow{2}{*}{\multicolumn{4}{|c|}{$\left(10^{-7}\right.$ photons $\left.\mathrm{cm}^{-2} \mathrm{~s}^{-1}\right)$}} & \multirow{2}{*}{\multicolumn{2}{|c|}{$(\mathrm{keV})$}} \\
\hline & & & & & & & & \\
\hline & & & (4) & (5) & (6) & (7) & (8) & (9) \\
\hline CNR-E & $-1.6 \pm 0.6$ & $0.50_{-0.38}^{+0.72}$ & $21 \pm 4$ & $6.9_{-3.0}^{+3.1}$ & $\begin{array}{l}1.8^{+1.8} \\
-1.5\end{array}$ & $0.0(<1.9)$ & $\begin{array}{l}2.3_{-0.8}^{+1.4} \\
\end{array}$ & $152 / 179$ \\
\hline CNR-SE & $1.2_{-1.3}^{+1.6}$ & $\begin{array}{l}12_{-10}^{+83} \\
\end{array}$ & $6.5_{-2.2}^{+2.4}$ & $0.34(<2.24)$ & $0.18(<1.40)$ & $0.61(<2.40)$ & $4.8_{-3.3}^{+19.6}$ & $97 / 119$ \\
\hline
\end{tabular}

Columns: (1) Subregion name. (2)-(3) Photon index and normalization of the power-law component. (4)-(7) Normalizations of the iron lines. (8) Equivalent width of the $6.4 \mathrm{keV} \mathrm{Fe} \mathrm{K \alpha}$ line. (9) $C$-statistic value over degrees of freedom.

SF largely contributes to it and complicates the analyses. The data above $7.5 \mathrm{keV}$ are filtered because of the low effective area, and in order to avoid uncertainty in edge of the AGN model, which is incorporated into spectral fits. Note that the pile-up effect is negligible $(<5 \%)$ in the two subregions (Section 4.3). Background spectra are estimated from a blank 50-arcsec radius circle located in the same CCD. Response files are generated using the CIAO tool specextract. We examine time variability within each observation based on the Gregory-Loredo algorithm ${ }^{\text {iv }}$, and find it to be insignificant. Thus, we average the individual spectra. We bin the spectra so that each energy bin has at least one count. In the same way as in Section 4.4, the best-fits are determined using the $C$-statistic technique on XSPEC. The spectra do not show significant variability between the two observations, and thus are fitted simultaneously with a single model to improve the SNR, without merging them.

Figure 3 shows the spectra folded by the response function. We uniformly fit a power-law plus four Gaussian functions (zgauss in XSPEC terminology), reproducing the $\mathrm{Fe} \mathrm{K} \alpha$ line at $6.40 \mathrm{keV}$, the $\mathrm{Fe} \mathrm{K} \beta$ line at $7.06 \mathrm{keV}$, the Fe XXV He $\alpha$ line at $6.68 \mathrm{keV}$, and the Fe XXVI Ly $\alpha$ line at $6.97 \mathrm{keV}$. The line widths are fixed at $\sigma=20 \mathrm{~km} \mathrm{~s}^{-1}$ or 0.4 $\mathrm{eV}$ by assuming that the iron atoms couple with molecules, whose velocities are roughly estimated from the ALMA data (Section 5.1.1). Note that even if the line widths are fixed at $0 \mathrm{eV}$, our conclusion is not affected. The AGN emission determined in Section 4.2.1 is further incorporated through response files produced by assuming a point source located in the AGN position. This component is fixed for simplicity. In summary, the free parameters are the normalization and photon index $(\Gamma)$ of the power-law component, and the line normalizations. The fit results are summarized in Table 4 . We significantly detect Fe $\mathrm{K} \alpha$ lines in the CNR-E and CNR-SE regions, whose EWs are $2.3_{-0.8}^{+1.4} \mathrm{keV}$ and $4.8_{-3.3}^{+19.6} \mathrm{keV}$, respectively. Such high EWs can be reproduced if a direct photoionizing X-ray source is not seen in the sightline and the reflected X-ray emission dominates the continuum (e.g., Terashima \& Wilson 2001;

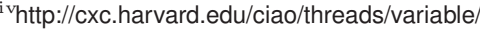

Nobukawa et al. 2010; Tanimoto et al. 2018).

Motivated by the above results, we further fit the spectra by replacing the power-law with a more physicallymotivated model of pexrav. The model calculates a reflected continuum spectrum from an optically thick neutral slab with a solid angle $(R \equiv \Omega / 2 \pi)$ irradiated by a cutoff power-law component (Magdziarz \& Zdziarski 1995). We fix the normalization, photon index, and cut-off energy of the incident power-law component to 0.7 photons at $\mathrm{keV}^{-1} \mathrm{~cm}^{-2} \mathrm{~s}^{-1}$ at $1 \mathrm{keV}, 2.31$, and $160 \mathrm{keV}$ based on the result of Arévalo et al. (2014). The inclination angle to the slab, which has little impact on results, is representatively fixed at $60^{\circ}$. The same power-law and inclination angle are adopted also in below models if required. Thus, the pexrav model has the only free parameter of the solid angle $(R)$. The best-fit models of CNR-E $(C$ statistics/d.o.f $=160 / 180)$ and CNR-SE ( $C$-statistics/d.o.f $=98 / 120)$ are obtained with $R=5.7_{-0.7}^{+0.8} \times 10^{-4}$ and $R=$ $2.4_{-0.9}^{+1.0} \times 10^{-4}$. Their EWs are estimated to be $4.1_{-1.1}^{+1.5} \mathrm{keV}$ and $2.6_{-1.5}^{+2.4} \mathrm{keV}$, respectively. Although the face values of the solid angles suggest very tiny reflection material, these can be interpreted as lower limits, given that the normalization of the power-law component may be suppressed by absorption. If we alternatively fix the solid angle to 1 and leave the power-law normalization free, we can get $4.0 \pm 0.5 \times 10^{-4}$ photons $\mathrm{keV}^{-1} \mathrm{~cm}^{-2} \mathrm{~s}^{-1}$ for CNR-E and $1.7 \pm 0.4 \times 10^{-4}$ photons $\mathrm{keV}^{-1} \mathrm{~cm}^{-2} \mathrm{~s}^{-1}$ for CNR-SE. We can also reproduce the spectra well using pexmon (Nandra et al. 2007) instead of pexrav. The model gives the sum of the continuum from the pexrav model (Magdziarz \& Zdziarski 1995), the fluorescence lines of Fe-K $\alpha$, Fe-K $\beta$, and $\mathrm{Ni}-\mathrm{K} \alpha$, and the Compton shoulder of the Fe-K $\alpha$ line, in a self-consistent way. The fitting returns almost the same solid angles $\left(R=7.6_{-0.7}^{+0.8} \times 10^{-4}\right.$ with $C$-statistics/d.o.f $=$ $200 / 182$ and $R=2.8 \pm 0.6 \times 10^{-4}$ with $C$-statistics/d.o.f $=101 / 122$ for CNR-E and CNR-SE, respectively). In the same manner as in the case of pexrav, we fit the spectra by fixing $R$ at 1 and allowing the normalization to vary, and obtain $5.3_{-0.5}^{+0.6} \times 10^{-4}$ photons $\mathrm{keV}^{-1} \mathrm{~cm}^{-2} \mathrm{~s}^{-1}$ for CNR-E and $1.9 \pm 0.4 \times 10^{-4}$ photons $\mathrm{keV}^{-1} \mathrm{~cm}^{-2} \mathrm{~s}^{-1}$ for CNR-SE. Finally, we fit the xillver model (García et al. 2013), a more complicated reflection spectral model than 
the pexmon model. That allows us to change the ionization state. In the fits, the normalization (an alternative parameter of the solid angle) and the ionization parameter $\left(\xi_{\mathrm{x}}\right)$ for X-ray (1-1000 Ry energy) emission (see Equation (10) in García et al. 2013 for its definition) are left as free parameters. In either region of interest, we obtain the lowest ionization parameter accepted by the model $\left(\xi_{\mathrm{x}}=1\right)$. This favors $\xi_{\mathrm{X}} \sim 0.2$, estimated by assuming gas at 60 pc distance from the nucleus being irradiated by X-ray emission with $\approx 3 \times 10^{42} \mathrm{erg} \mathrm{s}^{-1}$ through an medium with $N_{\mathrm{H}}=10^{23.9} \mathrm{~cm}^{-1}$ (i.e., optical depth $=1$ for X-rays at the K-edge energy of neutral iron). Thus, we suggest that the gas in the CNR-E and CNR-SE regions is irradiated by $\mathrm{X}$-ray emission from the central AGN.

\section{ALMA DATA ANALYSES}

\subsection{Reduction and Reprocessing of ALMA Data}

We reprocessed the ALMA data via the Common Astronomy Software Application (CASA) software package (McMullin et al. 2007) and adopted the same system versions as those used in the Quality Verification by the ALMA Regional Center. To extract signals from the molecular emission lines, we subtracted the continuum component from the data cube via the task uvcontsub. The continuum level was determined by fitting line-free channels with the first-order function. Then, we analyze the ALMA (a) and (b) datasets according to the scientific purpose of use.

We used the (a) dataset mainly to constrain the molecular gas properties through the molecular line ratios. The dataset was composed of two observations. We matched their spatial and velocity resolutions to extract the spectra under the same conditions as much as possible. We thus applied the clean task with uvcoverage $=13-250 k \lambda$ to match the $u v$-coverage. The range was covered by the two observations. The channels were binned such that the velocity resolution was $\approx 20 \mathrm{~km} \mathrm{~s}^{-1}: 20.3 \mathrm{~km}$ $\mathrm{s}^{-1}$ for $\operatorname{CO}(J=3-2), 17.8 \mathrm{~km} \mathrm{~s}^{-1}$ for $\operatorname{HCN}(J=3-2), 19.9$ $\mathrm{km} \mathrm{s}^{-1}$ for $\operatorname{HCN}(J=4-3), 17.7 \mathrm{~km} \mathrm{~s}^{-1}$ for $\mathrm{HCO}^{+}(J=3-$ $2)$, and $19.8 \mathrm{~km} \mathrm{~s}^{-1}$ for $\mathrm{HCO}^{+}(J=4-3)$. We adopted Briggs-weighting with robust $=0.5$ and gain $=0.1$ to produce images with high resolution and high sensitivity per beam. The primary beam image correction was made via impbcor. Finally, we applied the imsmooth task to match the beam sizes to the worst of $1.01 \times 1.37$ $\operatorname{arcsec}^{2}\left(\approx 20 \times 27 \mathrm{pc}^{2}\right)$ with $\mathrm{PA}=48.8$ degrees, which was achieved for the $\operatorname{HCN}(J=3-2)$ line. Note that the task was not applied for the $\mathrm{HCO}^{+}(J=3-2)$ line because of almost the same beam size (i.e., $\left.1.00 \times 1.37 \operatorname{arcsec}^{2}\right)$. The fi- nal continuum-subtracted data had RMS noise of $5.3 \mathrm{mJy}$ beam $^{-1}$ for $\mathrm{CO}(J=3-2), 2.5 \mathrm{mJy}^{b^{-1}}{ }^{-1}$ for $\operatorname{HCN}(J=3$ 2), $6.8 \mathrm{mJy} \mathrm{beam}^{-1}$ for $\mathrm{HCN}(J=4-3), 2.7 \mathrm{mJy}^{\text {beam }}{ }^{-1}$ for $\mathrm{HCO}^{+}(J=3-2)$, and $7.3 \mathrm{mJy}$ beam ${ }^{-1}$ for $\mathrm{HCO}^{+}(J=4-3)$. These values were estimated from the emission-free channels. For the $\operatorname{CO}(J=3-2)$ line, some negative signals were found around the line, likely due to the high brightness and sparse $u v$-coverage. Thus, to make the correction, we simply added the systematic error of $240 \mathrm{mJy}^{\text {beam }}{ }^{-1}$ when fitting the spectra.

Figure 4 shows the velocity-integrated intensity maps (Moment 0), intensity-weighted mean velocity maps (Moment 1), and intensity-weighted velocity dispersion maps (Moment 2) of the five lines. Smoothing was not conducted. The Moment 0 maps were made by calculating the 0th moment over the VLSR range of 200-650 $\mathrm{km} \mathrm{s}^{-1}$. The other moment maps were produced in the same VLSR range, but $10 \sigma$ clipping was further applied. Note that the Moment maps of the $\mathrm{CO}(J=3-2)$ line only take account of the statistical error.

In contrast to the (a) dataset, the (b) dataset was used to reveal the molecular gas distributions in detail by exploiting the high SNR. We produced the $\mathrm{HCO}^{+}(J=4-3)$ line data cube via the clean task using the same options adopted above. However, we increased the velocity resolution to $9.8 \mathrm{~km} \mathrm{~s}^{-1}$ so as not to miss the broad components. Setting a different velocity resolution does not largely affect our discussion. The primary beam correction was conducted using the impbcor task. Figure 5 shows the Moment 0, 1, and 2 maps of $\mathrm{HCO}^{+}(J=4-3)$. Except for the Moment 0 map, $10 \sigma$ clipping was applied. We adopted a wider channel range of VLSR $=150-700 \mathrm{~km}$ $\mathrm{s}^{-1}$ to cover broad but faint components. High RMS noise of $0.73 \mathrm{mJy}$ beam $^{-1}$ was achieved, while the beam size was $0.61 \times 0.59 \operatorname{arcsec}^{2}$ with $\mathrm{PA}=-1.25$. Note that the Moment 2 map shows a bit higher values than that created using the (a) dataset. We confirmed that line fluxes and velocity-integrated brightness temperatures used for discussion were however consistent between the (a) and (b) datasets.

\subsubsection{Basic Molecular Line Properties}

We constrain the basic quantities of the emission lines in the three subregions (Nucleus, CNR-E, and CNR$\mathrm{SE})$ through the spectra taken with a single synthesized beam $\left(\approx 1.01 \times 1.37 \operatorname{arcsec}^{2}\right)$ centered at each region (see Figure 5). The Gaussian functions are fitted as shown in Figure 6 . The best-fits are determined using the least chisquare method. We cannot achieve acceptable fits to the Nucleus spectra using a single Gaussian function. Thus, two functions are adopted. The spectra taken from CNR-E 

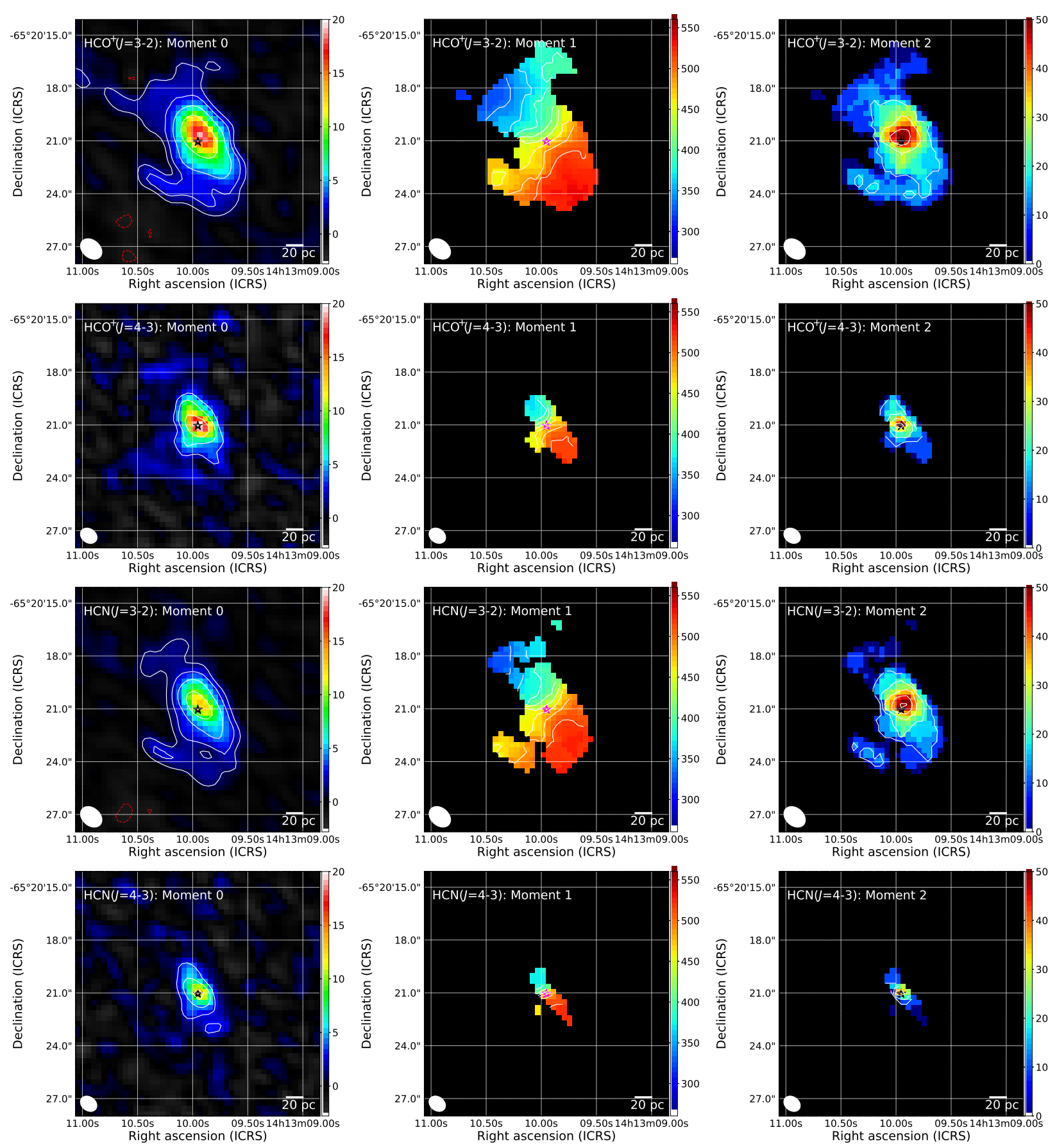

Fig. 4. (Left) Velocity-integrated intensity maps created using channels from VLSR $=200$ to $650 \mathrm{~km} \mathrm{~s}^{-1}$. The contour levels are $5 \sigma, 10 \sigma, 20 \sigma, 40 \sigma$, and $80 \sigma$, where $\sigma$ is $0.24 \mathrm{Jy}_{\text {beam }}^{-1} \mathrm{~km} \mathrm{~s}^{-1}, 0.61 \mathrm{Jy} \mathrm{beam}^{-1} \mathrm{~km} \mathrm{~s}^{-1}, 0.23 \mathrm{Jy} \mathrm{beam}{ }^{-1} \mathrm{~km} \mathrm{~s}^{-1}$, and $0.55 \mathrm{Jy}_{\text {beam }}{ }^{-1} \mathrm{~km} \mathrm{~s}^{-1}$ for $\mathrm{HCO}^{+}(J=3-2), \mathrm{HCO}^{+}(J=4-3), \mathrm{HCN}(J=3-2)$, and $\mathrm{HCN}(J=4-3)$, respectively. Negative signals at $-5 \sigma$ are also shown in red contours, if present. (Middle) Intensity-weighted mean velocity maps. The contours represent the VLSR with steps of $25 \mathrm{~km} \mathrm{~s}^{-1}$. (Right) Intensity-weighted velocity dispersion maps with the contours separated by $10 \mathrm{~km} \mathrm{~s}^{-1}$. The bottom-left filled ellipses represent beam sizes of $1.37 \times 1.00 \operatorname{arcsec}^{2}$ with $P A=49.2$ degrees, $1.02 \times 0.80$ $\operatorname{arcsec}^{2}$ with $\mathrm{PA}=54.9$ degrees, $1.37 \times 1.01 \mathrm{arcsec}^{2}$ with $\mathrm{PA}=48.8$ degrees, and $1.02 \times 0.79 \operatorname{arcsec}^{2}$ with $\mathrm{PA}=52.8$ degrees for $\mathrm{HCO}^{+}(J=3-2), \mathrm{HCO}^{+}(J=4-3), \mathrm{HCN}(J=3-2)$, and $\mathrm{HCN}(J=4-3)$, respectively. The SMBH position is denoted with the black (Moment 0 and 2) or magenta (Moment 1) star. 

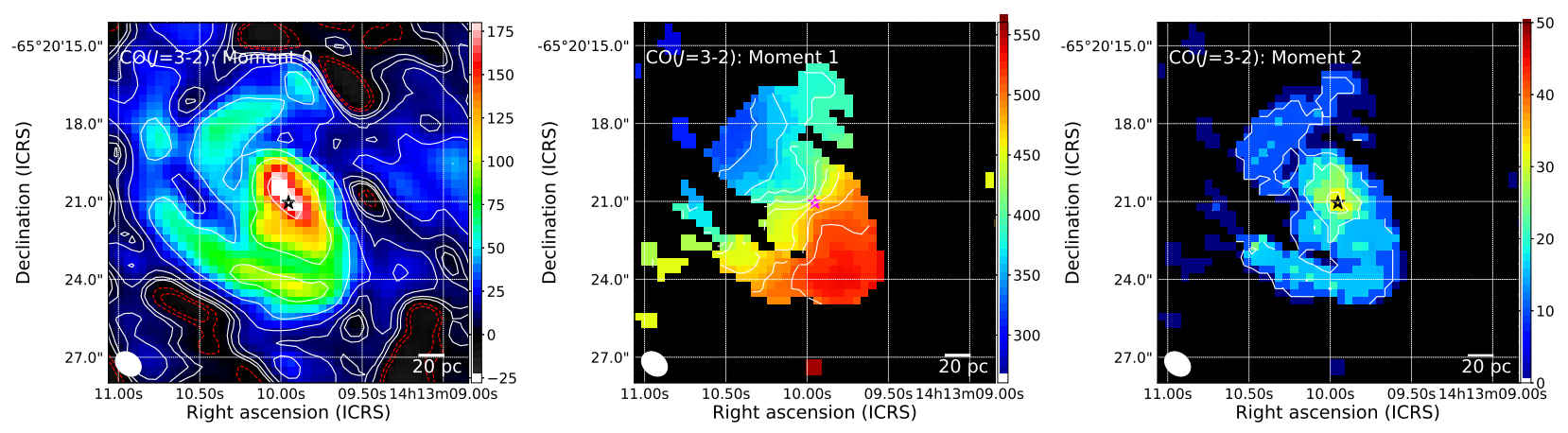

Fig. 4. (Left) Velocity-integrated intensity map of $\mathrm{CO}(J=3-2)$ created using channels from VLSR $=200$ to $650 \mathrm{~km} \mathrm{~s}^{-1}$. The white contour levels are $5 \sigma, 10 \sigma, 20 \sigma, 40 \sigma, 80 \sigma, 160 \sigma$, and $320 \sigma$, where $\sigma$ is $0.521 \mathrm{Jy} \mathrm{beam}^{-1} \mathrm{~km} \mathrm{~s}^{-1}$. Negative signals at $-10 \sigma$ and $-5 \sigma$ are also shown in red contours. (Middle) Intensity-weighted mean velocity map. The contours represent the VLSR with steps of $25 \mathrm{~km} \mathrm{~s}^{-1}$. (Right) Intensity-weighted velocity dispersion map with the contours separated by 10 $\mathrm{km} \mathrm{s}^{-1}$. The bottom-left filled ellipses represent a beam size of $1.09 \times 0.84 \operatorname{arcsec}^{2}$ with PA $=50.8$ degrees. The SMBH position is denoted with the black (Moment 0 and 2) or magenta (Moment 1) star.
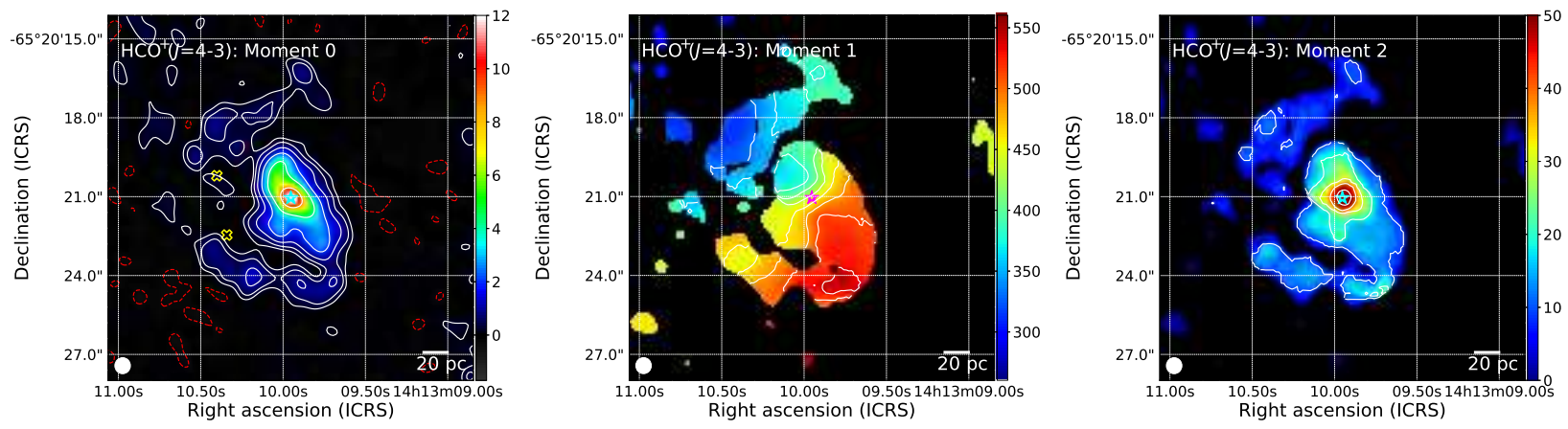

Fig. 5. (Left) Velocity-integrated intensity map of $\mathrm{HCO}^{+}(J=4-3)$ created using channels from VLSR $=150$ to $700 \mathrm{~km} \mathrm{~s}^{-1}$. The contour levels are $5 \sigma, 10 \sigma, 20 \sigma, 40 \sigma, 80 \sigma$, and $160 \sigma$, where $\sigma$ is $0.054 \mathrm{Jy} \mathrm{beam}^{-1} \mathrm{~km} \mathrm{~s}^{-1}$. Negative signals at $-5 \sigma$ are also shown in red contours. The spectra in Figure 6 were extracted using the synthesized beams centered at the yellow crosses, except for the Nucleus, the spectra of which are taken from the center (the cyan star). (Middle) Intensity-weighted mean velocity map. The contours represent the VLSR with steps of $25 \mathrm{~km} \mathrm{~s}^{-1}$. (Right) Intensity-weighted velocity dispersion map with the contours separated by $10 \mathrm{~km} \mathrm{~s}^{-1}$. The bottom-left filled ellipses represent a beam size of $0.61 \times 0.59$ arcsec ${ }^{2}$ with $\mathrm{PA}=-1.25$ degrees. The SMBH position is denoted with the cyan (Moment 0 and 2) or magenta (Moment 1 ) star.

and CNR-SE are well reproduced using a single Gaussian function. Because the $\operatorname{HCN}(J=4-3)$ lines in the CNR-E and CNR-SE regions are weak, we fix their velocity widths and line centers at those constrained at each $\operatorname{HCN}(J=3-2)$ line. Table 5 summarizes the parameters of the Gaussian functions, and the resultant velocity-integrated intensities and brightness temperatures.

\subsubsection{RADEX Non-LTE Modeling}

The physical and chemical properties of the molecular gas are constrained by comparing the observed molecular line ratios to those predicted by the non-local thermodynamic equilibrium (non-LTE) code of RADEX (van der Tak et al. 2007). We simulate 10 combinations of line ratios taken from the five lines for various kinetic temperatures $\left(T_{\text {kin }}\right)$, hydrogen densities $\left(n_{\mathrm{H} 2}\right)$, and hydrogen column densities $\left(N_{\mathrm{mol}}\right)$. A spherically, homogeneous geometry is assumed in the code. The molecular line transition rates are compiled from the Leiden Atomic and Molecular Database (LAMDA; Schöier et al. 2005). We cover the gas kinetic temperature within $T_{\text {kin }}=10-600 \mathrm{~K}$ for steps of $\Delta T_{\text {kin }}$ $=10 \mathrm{~K}$, the gas density within $\log n_{\mathrm{H} 2} / \mathrm{cm}^{-3}=2-7$ for steps of $\Delta \log n_{\mathrm{H} 2} / \mathrm{cm}^{-3}=0.5$, and the column density within $\log N_{\mathrm{H} 2} / \mathrm{cm}^{-2}=20-25$ for steps of $\Delta \log N_{\mathrm{H} 2} / \mathrm{cm}^{-2}$ $=0.5$. In addition, the $\mathrm{HCN}$ to $\mathrm{HCO}^{+}$abundance ratio $\left([\mathrm{HCN}] /\left[\mathrm{HCO}^{+}\right]\right)$is varied from 1 to 10 for steps of 1 by considering previous studies that indicated the enhanced HCN abundance in AGN host galaxies (e.g., Kohno 2005; 

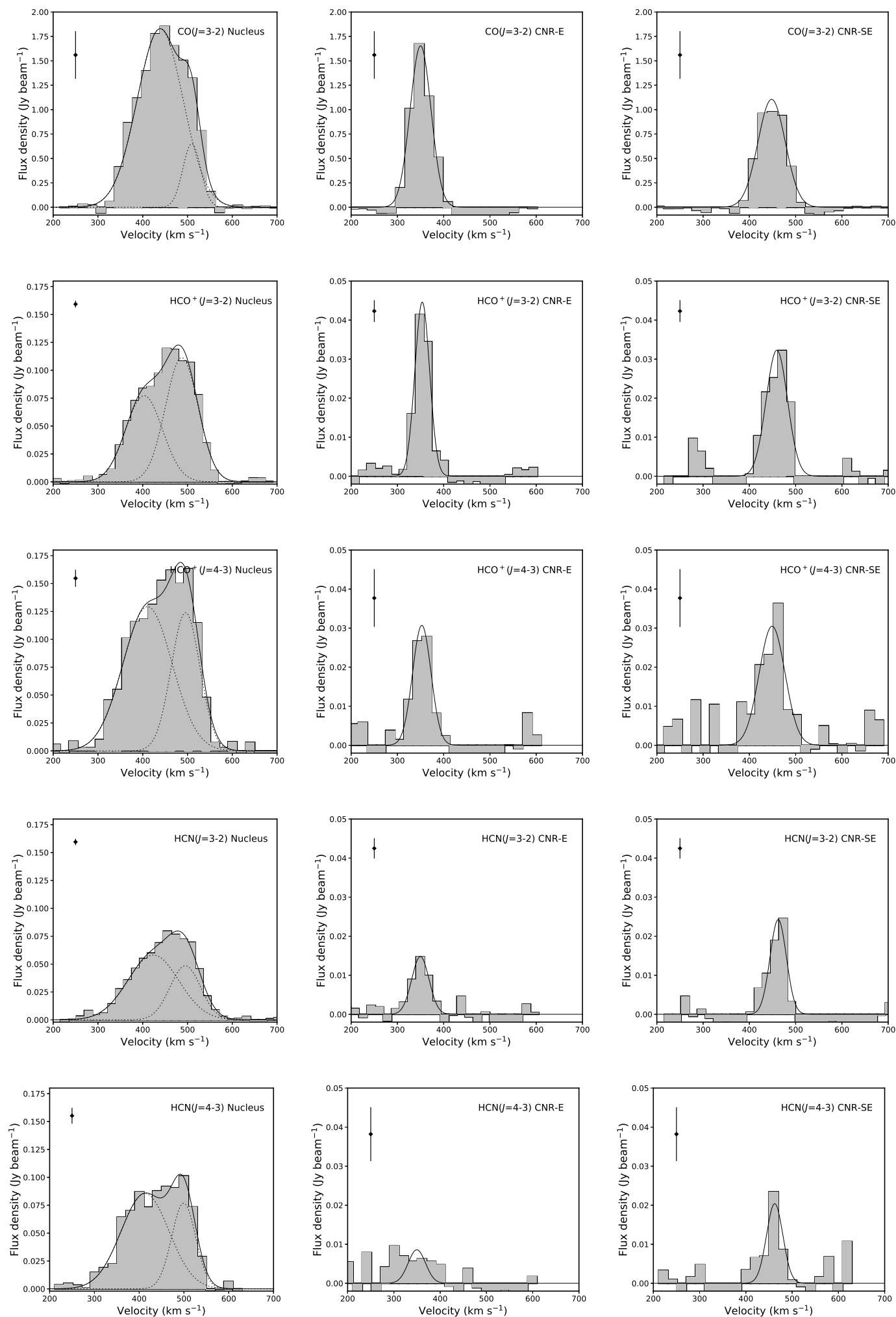

Fig. 6. Molecular emission lines of $\mathrm{CO}(J=3-2), \mathrm{HCO}^{+}(J=3-2), \mathrm{HCO}^{+}(J=4-3), \mathrm{HCN}(J=3-2)$, and $\mathrm{HCN}(J=4-3)$. From left to right, the figures present the spectra extracted from the Nucleus, CNR-E, and CNR-SE regions with a beam size of $\approx 1.01 \times 1.37 \operatorname{arcsec}^{2}\left(\approx 20 \times 27 \mathrm{pc}^{2}\right)$. Each scale bar in each top left corner corresponds to $1 \sigma$ error. In the Nucleus spectra, the two Gaussian functions and the total are plotted with dotted and solid lines, respectively. In the remaining spectra, each single Gaussian function is plotted with a solid line. 
Table 5. Molecular Line Properties

\begin{tabular}{|c|c|c|c|c|c|c|c|c|}
\hline $\begin{array}{c}\text { Molecular line } \\
\text { (1) }\end{array}$ & $\begin{array}{c}\nu_{\text {rest }} \\
(\mathrm{GHz}) \\
(2)\end{array}$ & $\begin{array}{c}\text { Region } \\
\text { (3) }\end{array}$ & $\begin{array}{c}a \\
\left(\mathrm{Jy}_{\mathrm{y}} \mathrm{beam}^{-1} \mathrm{~km} \mathrm{~s}^{-1}\right) \\
(4)\end{array}$ & $\begin{array}{c}\sigma \\
(\mathrm{km} \mathrm{s}-1) \\
(5)\end{array}$ & $\begin{array}{c}{ }^{v_{\mathrm{LSR}}}{ } \\
\left(\mathrm{km} \mathrm{s}^{-1}\right) \\
(6)\end{array}$ & $\begin{array}{c}\text { Peak flux } \\
\left(\mathrm{Jy} \mathrm{beam}^{-1}\right) \\
(7)\end{array}$ & $\begin{array}{c}I \\
\left(\mathrm{Jy}^{\prime} \text { beam }\right. \\
(8) \\
(8)\end{array}$ & $\begin{array}{c}I \\
\left(\mathrm{~K} \mathrm{~km} \mathrm{~s}^{-1}\right) \\
(9)\end{array}$ \\
\hline \multirow[t]{3}{*}{$\mathrm{CO}(J=3-2)$} & 345.796 & Nucleus $^{2}$ & $\begin{array}{c}32.6 \pm 13.6 \\
234 \pm 16\end{array}$ & $\begin{array}{l}20.0 \pm 4.4 \\
51.1 \pm 3.2\end{array}$ & $\begin{array}{l}510 \pm 3 \\
439 \pm 4\end{array}$ & $\begin{array}{c}0.649 \pm 0.307 \\
1.83 \pm 0.17\end{array}$ & $\begin{array}{c}13.9 \pm 7.2 \\
99.6 \pm 11.1 \\
113.4 \pm 13.2^{*}\end{array}$ & $\begin{array}{c}102 \pm 53 \\
736 \pm 82 \\
838 \pm 98^{*}\end{array}$ \\
\hline & & CNR-E $^{1}$ & $93.7 \pm 5.6$ & $22.6 \pm 1.6$ & $350 \pm 2$ & $1.65 \pm 0.15$ & $39.8 \pm 4.6$ & $294 \pm 34$ \\
\hline & & CNR-SE $^{1}$ & $81.6 \pm 3.3$ & $29.5 \pm 1.4$ & $448 \pm 1$ & $1.11 \pm 0.07$ & $34.7 \pm 2.7$ & $256 \pm 20$ \\
\hline \multirow[t]{3}{*}{$\mathrm{HCO}^{+}(J=3-2)$} & 267.558 & Nucleus $^{2}$ & $\begin{array}{l}7.99 \pm 2.00 \\
10.2 \pm 2.0\end{array}$ & $\begin{array}{l}41.3 \pm 6.3 \\
36.6 \pm 3.5\end{array}$ & $\begin{array}{c}403 \pm 11 \\
487 \pm 6\end{array}$ & $\begin{array}{l}0.077 \pm 0.023 \\
0.111 \pm 0.024\end{array}$ & $\begin{array}{r}3.40 \pm 1.13 \\
4.34 \pm 1.03 \\
7.73 \pm 1.52^{*}\end{array}$ & $\begin{array}{r}42.3 \pm 14.0 \\
54.0 \pm 12.8 \\
96.4 \pm 19.0^{*}\end{array}$ \\
\hline & & CNR-E $^{1}$ & $1.78 \pm 0.13$ & $15.9 \pm 1.3$ & $354 \pm 1$ & $0.045 \pm 0.005$ & $0.757 \pm 0.103$ & $9.43 \pm 1.28$ \\
\hline & & CNR-SE $^{1}$ & $1.88 \pm 0.26$ & $23.2 \pm 3.7$ & $459 \pm 4$ & $0.032 \pm 0.007$ & $0.797 \pm 0.212$ & $9.93 \pm 2.64$ \\
\hline \multirow[t]{3}{*}{$\mathrm{HCO}^{+}(J=4-3)$} & 356.734 & Nucleus $^{2}$ & $\begin{array}{c}17.8 \pm 4.1 \\
9.98 \pm 3.95\end{array}$ & $\begin{array}{l}54.9 \pm 9.3 \\
32.2 \pm 4.8\end{array}$ & $\begin{array}{l}411 \pm 14 \\
496 \pm 5\end{array}$ & $\begin{array}{l}0.129 \pm 0.037 \\
0.124 \pm 0.052\end{array}$ & $\begin{array}{l}7.56 \pm 2.52 \\
4.24 \pm 1.91 \\
11.8 \pm 3.2^{*}\end{array}$ & $\begin{array}{c}52.5 \pm 17.5 \\
29.4 \pm 13.2 \\
81.9 \pm 21.9^{*}\end{array}$ \\
\hline & & CNR-E $^{1}$ & $1.54 \pm 0.36$ & $19.9 \pm 5.4$ & $353 \pm 5$ & $0.031 \pm 0.011$ & $0.652 \pm 0.291$ & $4.53 \pm 2.02$ \\
\hline & & CNR-SE $^{1}$ & $2.14 \pm 0.38$ & $28.0 \pm 5.7$ & $449 \pm 6$ & $0.030 \pm 0.008$ & $0.908 \pm 0.309$ & $6.30 \pm 2.15$ \\
\hline \multirow[t]{3}{*}{$\operatorname{HCN}(J=3-2)$} & 265.886 & Nucleus $^{2}$ & $\begin{array}{l}8.31 \pm 2.77 \\
4.16 \pm 2.68\end{array}$ & $\begin{array}{l}57.0 \pm 9.9 \\
34.3 \pm 6.3\end{array}$ & $\begin{array}{c}424 \pm 20 \\
495 \pm 5\end{array}$ & $\begin{array}{l}0.058 \pm 0.022 \\
0.048 \pm 0.032\end{array}$ & $\begin{array}{c}3.53 \pm 1.46 \\
1.77 \pm 1.23 \\
5.30 \pm 1.91^{*}\end{array}$ & $\begin{array}{c}44.1 \pm 18.3 \\
22.1 \pm 15.4 \\
66.2 \pm 23.9^{*}\end{array}$ \\
\hline & & CNR-E ${ }^{1}$ & $0.694 \pm 0.126$ & $18.7 \pm 3.9$ & $350 \pm 4$ & $0.015 \pm 0.004$ & $0.295 \pm 0.102$ & $3.69 \pm 1.27$ \\
\hline & & CNR-SE $^{1}$ & $1.07 \pm 0.14$ & $17.6 \pm 2.6$ & $462 \pm 3$ & $0.024 \pm 0.005$ & $0.456 \pm 0.113$ & $5.70 \pm 1.41$ \\
\hline \multirow[t]{3}{*}{$\mathrm{HCN}(J=4-3)$} & 354.505 & Nucleus $^{2}$ & $\begin{array}{c}11.5 \pm 2.0 \\
5.17 \pm 1.85\end{array}$ & $\begin{array}{l}53.5 \pm 8.5 \\
26.9 \pm 4.7\end{array}$ & $\begin{array}{c}13 \pm 11 \\
499 \pm 4\end{array}$ & $\begin{array}{l}0.086 \pm 0.020 \\
0.077 \pm 0.031\end{array}$ & $\begin{array}{c}4.87 \pm 1.39 \\
2.20 \pm 0.96 \\
7.07 \pm 1.69^{*}\end{array}$ & $\begin{array}{c}34.2 \pm 9.8 \\
15.5 \pm 6.7 \\
49.7 \pm 11.8^{*}\end{array}$ \\
\hline & & CNR-E $^{1}$ & $0.403 \pm 0.217$ & $18.7^{\dagger}$ & $350^{\dagger}$ & $0.009 \pm 0.005$ & $0.171 \pm 0.092$ & $1.20 \pm 0.65$ \\
\hline & & CNR-SE $^{1}$ & $0.902 \pm 0.174$ & $17.6^{\dagger}$ & $462^{\dagger}$ & $0.020 \pm 0.004$ & $0.383 \pm 0.074$ & $2.69 \pm 0.52$ \\
\hline
\end{tabular}

Notes.

Columns: (1) Molecular line name. (2) Rest frequency. (3) Subregion name. The exact positions are listed in Table 3 and are marked in Figure 5. Here, the superscripts represent the number of the Gaussian function(s) used to fit each spectrum. (4)-(6) Parameters defined in the Gaussian function of $a / \sqrt{2 \pi \sigma^{2}} \exp \left\{-\left(v-v_{\mathrm{LSR}}\right)^{2} / 2 \sigma^{2}\right\}$. (7)-(8) Peak flux density and velocityintegrated flux density. (9) Velocity-integrated brightness temperature, derived from (8) by adopting a beam size of $1.01 \times 1.37$ $\operatorname{arcsec}^{2}$, except for the $\mathrm{HCO}^{+}(J=3-2)$ line, for which the $1.00 \times 1.37 \operatorname{arcsec}^{2}$ beam is adopted. Note that all estimates are based on the spectra binned at $\approx 20 \mathrm{~km} \mathrm{~s}^{-1}$ resolutions and corrected for the primary beam attenuation. * These values take account of the two Gaussian functions. † These values are fixed at those constrained in each $\operatorname{HCN}(J=3-2)$ line.

Table 6. Molecular Line Ratios

\begin{tabular}{cccccc}
\hline \hline Region & $\frac{\mathrm{HCO}^{+}(J=3-2)}{\mathrm{CO}(J=3-2)}$ & $\frac{\mathrm{HCO}^{+}(J=4-3)}{\mathrm{CO}(J=3-2)}$ & $\frac{\mathrm{HCN}(J=3-2)}{\mathrm{CO}(J=3-2)}$ & $\frac{\mathrm{HCN}(J=4-3)}{\mathrm{CO}(J=3-2)}$ & $\frac{\mathrm{HCO}^{+}(J=4-3)}{\mathrm{HCO}^{+}(J=3-2)}$ \\
& $(2)$ & $(3)$ & $(4)$ & $(5)$ & $(6)$ \\
& $\frac{\mathrm{HCN}^{+}(J=3-2)}{\mathrm{HCO}^{+}(J=3-2)}$ & $\frac{\mathrm{HCN}(J=4-3)}{\mathrm{HCO}^{+}(J=3-2)}$ & $\frac{\mathrm{HCO}^{+}(J=4-3)}{\mathrm{HCN}(J=3-2)}$ & $\frac{\mathrm{HCN}(J=4-3)}{\mathrm{HCN}(J=3-2)}$ & $\frac{\left.\mathrm{HCN}^{+} J=4-3\right)}{\mathrm{HCO}^{+}(J=4-3)}$ \\
& $(7)$ & $(8)$ & $(9)$ & $(10)$ & $(11)$ \\
\hline Nucleus & $0.0527 \pm 0.0142$ & $0.107 \pm 0.031$ & $0.0359 \pm 0.0145$ & $0.0639 \pm 0.0170$ & $2.03 \pm 0.74$ \\
& $0.681 \pm 0.280$ & $1.21 \pm 0.41$ & $2.99 \pm 1.47$ & $1.78 \pm 0.81$ & $0.596 \pm 0.214$ \\
\hline CNR-E & $0.0147 \pm 0.0026$ & $0.0169 \pm 0.0078$ & $0.0057 \pm 0.0021$ & $0.0044 \pm 0.0024$ & $1.15 \pm 0.54$ \\
& $0.387 \pm 0.144$ & $0.299 \pm 0.166$ & $2.97 \pm 1.67$ & $0.773 \pm 0.494$ & $0.261 \pm 0.182$ \\
\hline CNR-SE & $0.0178 \pm 0.0055$ & $0.0270 \pm 0.0094$ & $0.0101 \pm 0.0030$ & $0.0113 \pm 0.0024$ & $1.52 \pm 0.69$ \\
& $0.569 \pm 0.207$ & $0.637 \pm 0.228$ & $2.67 \pm 1.19$ & $1.12 \pm 0.39$ & $0.419 \pm 0.164$ \\
\hline
\end{tabular}

\section{Notes.}

Columns: (1) Subregion name. (2)-(11) Line ratios derived from fluxes in units of $\mathrm{erg} \mathrm{cm}^{-2} \mathrm{~s}^{-1}$. The absolute flux uncertainty (10\%) was taken into account if required. 
Izumi et al. 2013, 2016). For the other abundance ratios, we adopt canonical values of $[\mathrm{CO}] /\left[\mathrm{H}_{2}\right]=6 \times 10^{-5}$ and $\left[\mathrm{HCO}^{+}\right] /\left[\mathrm{H}_{2}\right]=8 \times 10^{-9}$, equivalent to those observed in galactic molecular clouds (Blake et al. 1987). We confirm that even if we vary the abundances to $[\mathrm{CO}] /\left[\mathrm{H}_{2}\right]=$ $5 \times 10^{-5}$ and $\left[\mathrm{HCO}^{+}\right] /\left[\mathrm{H}_{2}\right]=2 \times 10^{-9}$, estimated in other galactic molecular clouds (Blake et al. 1987), our conclusion in Section 6.3 is not strongly affected. The line velocities are fixed at the average $3 \sigma$ width of the five lines: $330 \mathrm{~km} \mathrm{~s}^{-1}, 120 \mathrm{~km} \mathrm{~s}^{-1}$, and $140 \mathrm{~km} \mathrm{~s}^{-1}$ for the Nucleus, CNR-E and CNR-SE regions, respectively. Our calculation also takes account of the blackbody radiation with $T_{\mathrm{bg}}=2.73 \mathrm{~K}$ as the uniform background emission. In summary, the free parameters are $T_{\text {kin }}, n_{\mathrm{H} 2}, N_{\mathrm{mol}}$, and $[\mathrm{HCN}] /\left[\mathrm{HCO}^{+}\right]$. These parameters are constrained by the chi-square method. The line ratios are calculated using fluxes in units of erg $\mathrm{cm}^{-2} \mathrm{~s}^{-1}$. Those observed are listed in Table 6 . The fitting results are summarized in Table 7.

\section{DISCUSSION}

\subsection{Spatial Comparison between Fe $\mathrm{K} \alpha$ Line and Molecular Emission Line}

Figure 7 shows the spatial distributions of the $6.4 \mathrm{keV}$ Fe $\mathrm{K} \alpha$ and $\mathrm{HCO}^{+}(J=4-3)$ lines at $\sim 0.5 \operatorname{arcsec}(\approx 10 \mathrm{pc})$ resolution. The most interesting feature is the spatial anti-correlation between the two emission lines. The iron line traces the gas irradiated by the hard X-rays from the AGN (see Section 4.4), and it is irrelevant whether the gas is in the molecular or atomic phase. In addition, the line is effectively emitted when X-rays propagate into the dense gas given the low cross-section. The $\mathrm{HCO}^{+}(J=4-3)$ line may also become luminous in such dense gas regions if abundant molecular gas is available as well, but is faint in regions with bright Fe K $\alpha$ line emission. The molecular emission seems to be brighter rather in the outer side. Thus, this anti-correlation indicates that molecules are dissociated into atoms in the proximity of the AGN due to the harsh X-ray radiation. Then, outer surfaces of the CNR-E and CNR-SE regions may correspond to the atomic-to-molecular gas transition boundary (right panel of Figure 7) within which photoelectrons produced by X-rays can greatly dissociate molecules. Although the Nucleus region is apparently closest to the central AGN, an amount of molecular gas is present. This is likely because we observe the molecular gas that is located far enough away from the central engine not to be effectively dissociated into atomic gas, like those found outside the other subregions. High inclination angles $\left(\sim 70^{\circ}\right)$ of the host galaxy as well as the molecular gas around the center, kinematically modeled by Izumi et al. (2018), support this idea.

\subsection{Diagram of Molecular Gas Line Ratios}

Based on the velocity-integrated temperature ratios, we briefly discuss molecular gas properties in the subregions. We can focus on the AGN effect rather than that of the $\mathrm{SF}$, given that our spectra were taken in the proximity of the nucleus at the $20 \mathrm{pc}$ scale and the SF is not influential due to the low SFR of $0.1 M_{\odot} \mathrm{yr}^{-1}$ in the central $75 \mathrm{pc}$ (Esquej et al. 2014).

Figure 8 shows a diagram of the $\operatorname{HCN}(J=4-$ $3) / \mathrm{HCN}(J=3-2)$ and $\mathrm{HCO}^{+}(J=4-3) / \mathrm{HCO}^{+}(J=3-2)$ ratios. Note that the ratios take into account an absolute flux uncertainty of $10 \%$. The Nucleus region shows the highest values in both ratios. The same diagram was made by Imanishi et al. (2018), who compiled AGN host and starburst galaxies. As judged from their Figure 26, the ratios of Circinus are low and comparable to those of starburst galaxies. NGC 7469, an unobscured AGN, also shows similar, low line ratios. If an AGN is able to heat the surrounding gas more effectively via X-ray emission rather than SF via UV emission, elevated line ratios would be expected for the AGN host galaxy. However, this was not observed. A possible cause is molecular gas dissociation, as discussed in Section 6.1. Indeed, if the molecular gas density is lower than the critical densities $\left(\sim 10^{6-7} \mathrm{~cm}^{-3}\right)$, the excitation level can become not so sensitive to the kinetic temperature or heating. At last, we note that the slightly higher $\mathrm{HCO}^{+}$line ratios than the $\mathrm{HCN}$ ones probably reflect that $\mathrm{HCO}^{+}$can be more easily excited due to a lower critical density than that of HCN (e.g., Greve et al. 2009).

Figure 9 shows a scatter plot between the HCN and $\mathrm{HCO}^{+}$line ratios at $J=4-3$ and $J=3-2$. These ratios have been studied extensively (e.g., Kohno 2005; Imanishi et al. 2010; Imanishi \& Nakanishi 2014; Izumi et al. 2013; Izumi et al. 2016; García-Burillo et al. 2014), and several studies have reported enhanced HCN emission in AGN host galaxies. Thus, questions remain as to whether AGN can be probed from the HCN line. Using data with high spatial resolution, Izumi et al. (2016) showed that AGN host galaxies may have $\mathrm{HCN}(J=4-3) / \mathrm{HCO}^{+}(J=4-3) \gtrsim 1$ (see also Viti et al. 2014; García-Burillo et al. 2014). However, Circinus has significantly lower values, implying that it may be difficult to identify all AGN based on HCN emission. As argued by Izumi et al. (2016), the $\operatorname{HCN}(J=4$ $3) / \mathrm{HCO}^{+}(J=4-3)$ ratio does not correlate with AGN Xray luminosity. Thus, an alternative mechanism, such as mechanical energy inputs by AGN jets/outflows or cosmicrays, would be more influential regarding the $\mathrm{HCN}$ en- 
Table 7. Physical Parameters of Molecular Gas

\begin{tabular}{cccccc}
\hline \hline Region & $\log N_{\mathrm{H}_{2}} / \mathrm{cm}^{-2}$ & $\log n\left(\mathrm{H}_{2}\right) / \mathrm{cm}^{-3}$ & $\begin{array}{c}T_{\mathrm{k}} \\
(\mathrm{K})\end{array}$ & {$[\mathrm{HCN}] /\left[\mathrm{HCO}^{+}\right]$} & $\chi^{2}$ \\
& & & $(4)$ & $(5)$ & $(6)$ \\
\hline Nucleus & $24.5_{-0.0}^{+0.5}$ & $5.0_{-0.5}^{+0.0}$ & $290_{-100}^{+110}$ & $3 \pm 0$ & 0.45 \\
CNR-E & $24.5_{-4.5}^{+0.5}$ & $3.5_{-0.5}^{+1.5}$ & $200_{-150}^{+130}$ & $2 \pm 1$ & 0.71 \\
CNR-SE & $23.5_{-0.0}^{+1.5}$ & $4.5_{-1.5}^{+0.0}$ & $130_{-50}^{+270}$ & $4_{-2}^{+1}$ & 0.42 \\
\hline
\end{tabular}

Notes.

Columns: (1) Subregion name. (2) Molecular hydrogen gas column density.

(3) Molecular hydrogen gas density. (4) Kinetic temperature. (5) Abundance

ratio between the $\mathrm{HCN}$ and $\mathrm{HCO}^{+}$molecules. (6) Chi-square value.
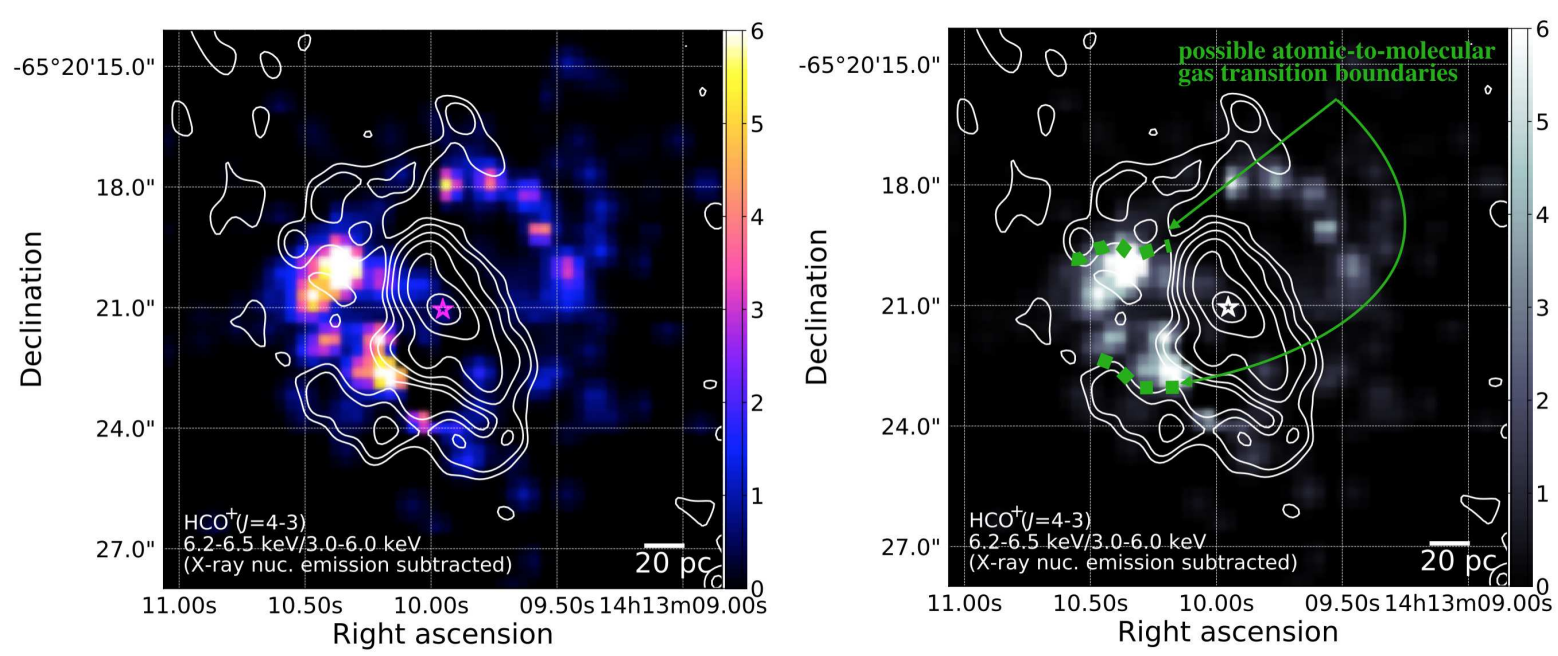

Fig. 7. (Left) Color-coded ratio between the 6.2-6.5 keV and 3.0-6.0 keV images, corresponding to a proxy of the Fe $\mathrm{K} \alpha$ line equivalent width, and the $\mathrm{HCO}^{+}(J=4-3)$ velocity-integrated intensity map (white contour). (Right) The same figure as in the left panel, except for the use of color and the illustration that represents possible atomic to molecular gas transition boundaries (green dashed lines). The magenta and white stars are located at the SMBH position.

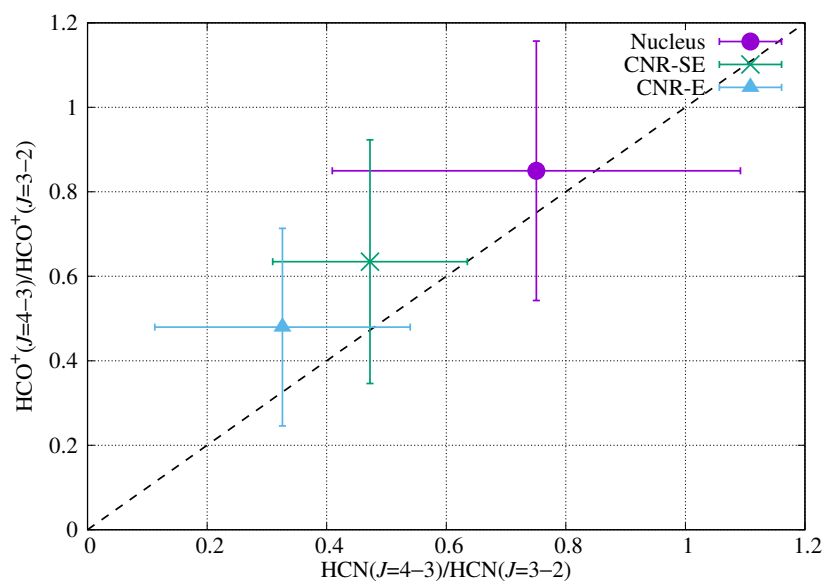

Fig. 8. Ratio of $\mathrm{HCN}(J=4-3)$ to $\mathrm{HCN}(J=3-2)$ versus that of $\mathrm{HCO}^{+}(J=4-3)$ to $\mathrm{HCO}^{+}(J=3-2)$ on the velocity-integrated brightness temperature scale. The dashed line represents the one-to-one line.

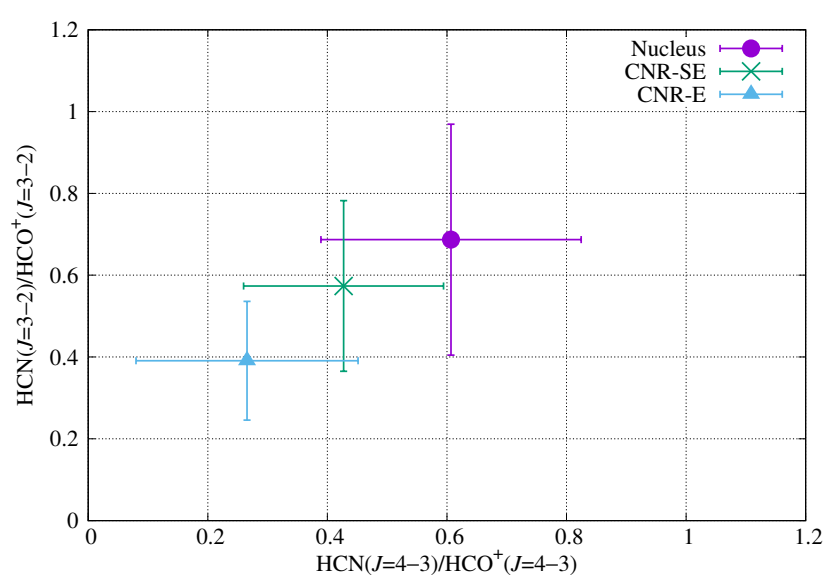

Fig. 9. Ratio of $\mathrm{HCN}(J=4-3)$ to $\mathrm{HCO}^{+}(J=4-3)$ versus that of $\mathrm{HCN}(J=3-2)$ to $\mathrm{HCO}^{+}(J=3-2)$ on the velocity-integrated brightness temperature scale. 
hancement. No report on such a signature for Circinus is consistent with this idea.

\subsection{XDR Model}

We quantitatively discuss the gas in the three subregions along with the XDR model, developed by Maloney et al. (1996). The gas-phase abundance of $\mathrm{C}$ and $\mathrm{O}$ with respect to $H$ is assumed to be $3.0 \times 10^{-4}$ and $5.0 \times 10^{-4}$, respectively. The X-ray spectrum incident on the medium is made up of single power-law emission. A key parameter for the discussion is the effective ionization parameter, which determines the fractional abundances of atomic and molecular gas species (see their Figure 3). The parameter is calculated as

$\xi_{\text {eff }} \simeq 0.1 \frac{L_{\mathrm{X}, 44}}{n_{\mathrm{H}_{2}, 4} R_{100}^{2} N_{\mathrm{H}, 22}^{\alpha}}$,

where $L_{\mathrm{X}, 44}, n_{\mathrm{H}_{2}, 4}, R_{100}, N_{\mathrm{H}, 22}$, and $\alpha$ represents the $1-$ $100 \mathrm{keV}$ luminosity in units of $10^{44} \mathrm{erg} \mathrm{s}^{-1}$, the hydrogen molecular gas density in units of $10^{4} \mathrm{~cm}^{-3}$, the distance from the X-ray source in units of $100 \mathrm{pc}$, the hydrogen column density of the gas that attenuates the incident $\mathrm{X}$-ray flux in units of $10^{22} \mathrm{~cm}^{-2}$, and an X-ray photon index-dependent value, respectively. The last factor $(\alpha)$ is specifically expressed as $\alpha=(\Gamma+2 / 3) /(8 / 3)$, and takes into account that softer spectra are more heavily absorbed. As canonical X-ray parameters for discussion, we adopt the $1-$ $100 \mathrm{keV}$ luminosity $\left(L_{1-100 \mathrm{keV}}\right)$ of $1.3 \times 10^{43} \mathrm{erg} \mathrm{s}^{-1}$ and the photon index of 2.31, derived from the NuSTAR data (Arévalo et al. 2014). The photon index corresponds to $\alpha \approx 1.1$. As detailed below, we can draw the same conclusion based on estimates from other hard X-ray observatories of BeppoSAX, INTEGRAL, and Suzaku (Soldi et al. 2005; Yang et al. 2009).

The molecular gas density has been derived from the non-LTE analyses (Section 5.1.2). The distances from the center to the CNR-E and CNR-SE regions were set to $62 \mathrm{pc}$ and $60 \mathrm{pc}$, respectively. The distance appropriate for the Nucleus region is difficult to determine due to the sightline. We here representatively consider $R>60 \mathrm{pc}$. This is based on the discussion in Section 6.1 that the Nucleus molecule we observe is equivalent to those seen outside the other subregions ( $\gtrsim 60 \mathrm{pc}$ ). Here, it may be stressed that robust constraint on the plausible atomic to molecular transition boundary is essential to calculate $\xi_{\text {eff }}$, and is achieved by exploiting the high spatial resolution $(\approx$ 0.5 arcsec) data taken by ALMA and Chandra. Finally, we assume $\log N_{\mathrm{H}} / \mathrm{cm}^{-2}=23.9$, where the medium becomes optically thick for the $7.1 \mathrm{keV}$ photon, the K-edge of neutral iron. This assumption is reasonable given that such photons are used up in the subregions, except for the
Table 8. Effective ionization parameters

\begin{tabular}{lc}
\hline \hline $\begin{array}{c}\text { Region } \\
(1)\end{array}$ & $\begin{array}{c}\log \xi_{\text {eff }} \\
(2)\end{array}$ \\
\hline Nucleus & {$[<-4.0]$} \\
CNR-E & {$[-4.6 \sim-2.6]$} \\
CNR-SE & {$[-4.0 \sim-2.5]$} \\
Notes. & \\
Columns: (1) Subregion name. & $(2)$ \\
Effective ionization parameters de- \\
fined in Equation 1.
\end{tabular}

Nucleus, and the fluorescent line is not bright beyond them (Figure 7).

As summarized in Table 8, the ionization parameters in the CNR-E and CNR-SE regions are $\log \xi_{\text {eff }}=-4.6 \sim-2.6$ and $-4.0 \sim-2.5$, respectively. These are consistent with $\log \xi_{\text {eff }} \approx-3.0$, above which the XDR model predicts a large fraction of the molecular hydrogen to be dissociated into atomic hydrogen. The low ionization parameter of $\log \xi_{\text {eff }}<-4.0$ in the Nucleus is consistent with a moderate amount of the molecular gas still remaining therein (Figure 7). Seemingly, this is contrary to the prediction of the XDR model, that is, the increase of the ionization parameter with decreasing distance. As already discussed (Section 6.1), this apparent discrepancy can be resolved by considering that the molecular gas in the Nucleus is located far away from the central source and makes it difficult to confirm the model prediction. Even if the abundances different from the default values are assumed (see Section 5.1.2), our conclusion does not change within $2 \sigma$. $\mathrm{X}$-ray parameters we adopt are also the factors of uncertainty. Soldi et al. (2005) derived $L_{1-100 \mathrm{keV}}=1.6 \times 10^{42}$ with $\Gamma=1.8$ and $L_{1-100 \mathrm{keV}}=1.4 \times 10^{42}$ with $\Gamma=1.5$ by fitting INTEGRAL/ISGRI+SPI and BeppoSAX/PDS spectra, respectively. Also, Yang et al. (2009) reproduced Suzaku/XIS+HXD broadband X-ray spectra with $L_{1-100 \mathrm{keV}}=3.8 \times 10^{42}$ and $\Gamma=1.58$. In either case, low ionization parameters of $\log \xi_{\text {eff }} \approx-3$ are derived, however. Thus, generally the spatial anti-correlation between the $\mathrm{Fe}$ $\mathrm{K} \alpha$ and molecular gas emission lines can be interpreted as the molecular gas dissociation by the AGN X-ray radiation.

Our study provides new insights into the nuclear structure of Circinus as well as highly obscured Compton-thick objects. As proposed by Izumi et al. (2018), Circinus is expected to have a radiation-driven fountain torus within $\sim 10$ pc (e.g., Wada 2012 and Wada et al. 2018). Because the model has low molecular/atomic gas densities in its polar regions, emission from the vicinity of the SMBH should preferably escape through them. Optical ionization cones 
in the northwest direction (Marconi et al. 1994) is likely the result, while its counterpart is invisible due to dust lanes even if any. This time, the Fe $\mathrm{K} \alpha$ emission is detected in the corresponding region, and supports the presence of a kind of the putative narrow line region behind the dust. Another interesting point comes from the fact that the Fe K $\alpha$ emission is effectively produced in regions with high column densities $\left(N_{\mathrm{H}} \sim 10^{24} \mathrm{~cm}^{-2}\right)$. The Fe K $\alpha$ line at $\sim 60 \mathrm{pc}$ suggests that a fraction of the X-ray emission that escaped from the torus is blocked by CNR-scale thick structures. That is, such CNR-scale material has potential to strongly contribute to obscuration if it is located in the sightline.

\subsection{SF in X-ray-irradiated Regions}

We discuss the subsequent impact of $\mathrm{X}$-ray radiation on $\mathrm{SF}$. A suppressed SFR is expected in the X-ray-irradiated regions, given the positive correlation between the molecular gas and SFR surface densities (e.g., Kennicutt et al. 2007; Bigiel et al. 2008). Roche et al. (2006) reported a Gemini South/T-ReCS long-slit spectroscopy observation of Circinus at 0.27 -arcsec resolution with the slit width of 0.36 arcsec (Telesco et al. 1998). They observed the $11.3 \mu \mathrm{m}$ polycyclic aromatic hydrocarbon (PAH) emission, whose luminosity is a proxy of the SFR. The less dust extincted PAH emission is convenient for tracing SFs, even in the dusty nuclear region. The T-ReCS slit was placed at two PAs of $100^{\circ}$ and $10^{\circ}$. The former roughly corresponds to the orientation towards the CNR-SE region from the Nucleus (see Figure 1 of Roche et al. 2006 for the slit configuration). They detected significant PAH emission around the CNR-SE region. However, they concluded that the emission originated from the foreground against the dust obscuration, and were not associated with the ionization cone behind the dust. Hence, the SF may be inactive in the CNR-SE region, and the X-ray-irradiated region as well. This is consistent with expectations involving molecular gas dissociation. In the case of Circinus, the X-ray effect is limited within $\sim 100 \mathrm{pc}$, but if the Xray $(1-100 \mathrm{keV})$ luminosity reaches $10^{45} \mathrm{erg} \mathrm{s}^{-1}$, like the most luminous AGN, the size could be $\sim 1 \mathrm{kpc}$ where $\log n_{\mathrm{H} 2} / \mathrm{cm}^{-3}=3$ and $\log \xi_{\text {eff }}=-3$ are assumed. Thus, in this case, the X-ray emission may have non-negligible impact, even on galaxy-scale SF.

\section{SUMMARY}

Our main aim was to understand the effects of AGN X-ray emission on gas. Using Chandra and ALMA, we investigated the physical and chemical conditions of the gas in the central $\sim 100$ pc of Circinus at 0.5 arcsec, $\approx 10$ pc resolution. The high penetrating power of X-ray and submm/mm wavelengths, covered by Chandra and ALMA respectively, is highly suited to study of the nuclear dense gas region with a small bias against the absorption and dust extinction. The $6.4 \mathrm{keV} \mathrm{Fe} \mathrm{K} \alpha$ line was used to map the X-ray-irradiated gas irrespective of its molecular and atomic phases. The map was compared to that of the molecular $\mathrm{HCO}^{+}(J=4-3)$ emission line (Figure 7 ).

Our results indicate that molecular gas emission is faint in regions with bright $\mathrm{Fe} \mathrm{K} \alpha$ line emission and bright in the outer regions. These results imply that the molecular gas close to the AGN becomes dissociated, and on the far side, atomic-to-molecular gas transition boundaries form. We have quantitatively discussed this possibility according to the XDR model developed by Maloney (1999). The effective ionization parameter (Equation 1), which characterizes the fractional abundance of molecular and atomic gas species, was constrained in each subregion (Nucleus, CNR-E and CNR-SE). Those estimated in the CNR-E and CNR-SE regions were high enough to support the idea. Furthermore, we have discussed the X-ray irradiation effect on $\mathrm{SF}$; the inactive $\mathrm{SF}$ in $\mathrm{X}$-ray-irradiated regions may indicate that it consequently suppresses $\mathrm{SF}$, particularly in the proximity of AGN.

\section{Acknowledgments}

Part of this work was financially supported by the Grantin-Aid for JSPS Fellows for young researchers (T.K.). T.K. was supported by the ALMA Japan Research Grant of NAOJ Chile Observatory, NAOJ-ALMA-202. T.I. and M.I. are supported by JSPS KAKENHI grant numbers $17 \mathrm{~K} 14247$ and 15K05030, respectively. The scientific results reported in this article are based on data obtained from the Chandra Data Archive. This research has made use of software provided by the Chandra X-ray Center (CXC) in the application packages CIAO. This paper makes use of the following ALMA data: ADS/JAO.ALMA\#2015.1.01286.S and ADS/JAO.ALMA\#2016.1.01613.S. ALMA is a partnership of ESO (representing its member states), NSF (USA) and NINS (Japan), together with NRC (Canada) and NSC and ASIAA (Taiwan) and KASI (Republic of Korea), in cooperation with the Republic of Chile. The Joint ALMA Observatory is operated by ESO, AUI/NRAO and NAOJ. We appreciate the JVO portal (http://jvo.nao.ac.jp/portal/) operated by ADC/NAOJ for the quick look of the ALMA archive data. This research made use of APLpy, an open-source plotting package for Python (Robitaille \& Bressert 2012).

\section{Appendix A Supplemental Chandra data}

In Section 4.3, we have created the X-ray images from the ObsID $=12823$ and 12824 data, obtained without the grat- 
ing. Here, we try to increase the SNR by taking account of 0th order images, and the data obtained for SN 1996cr, $\sim 20$ arcsec away from Circinus. Table A summarizes our data set here. Some of the data are excluded mainly because of the emergence of a point source around the CNR-SE region or very short exposures ( $<$ several ksec). Figure A, created from the ObsID $=10225$ data, shows the source, making the analysis and discussion more complex. In summary, the total exposure increases by $214 \mathrm{ksec}$, or effectively by $\sim 132 \mathrm{ksec}$ at $6.5 \mathrm{keV}$ given the $\sim 60 \%$ reduced effective areav ${ }^{\mathrm{v}}$. The $6.2-6.5 \mathrm{keV}$-to-3.0-6.0 keV image ratio is calculated in the same manner as in Section 4.3, and its image is compared with the intensity map of the $\mathrm{HCO}^{+}(J=4-3)$ line in Figure B. The result suggests that particularly in the CNR-E region we can still see the anticorrelation, while in the CNR-SE region it seems to be not as clear as in Figure 7. The latter might be because we selectively exclude the data that have signals in CNR-SE in order to avoid the contamination from the point source. It would be however accepted that the molecular line is generally faint in regions with bright $\mathrm{Fe}-\mathrm{K} \alpha$ emission.

We finally mention the region around RA, Decl. = $14 \mathrm{~h} 13 \mathrm{~m} 09.40 \mathrm{~s},-65 \mathrm{~d} 20 \mathrm{~m} 22 \mathrm{~s}$, where the Fe-K $\alpha$ line with a moderately high EW $(\sim 1 \mathrm{keV})$ can be detected as inferred from Figure B. Therein, the molecular lines seem to be very faint (it is clear particularly in the $\mathrm{CO}(J=3$ 2) Moment 0 map), consistent with a decreased molecular density. It is however difficult to draw a robust conclusion in the same way as in Section 6.3, because of the faintness Hence, we do not discuss this further.

\section{References}

Arévalo, P., Bauer, F. E., Puccetti, S., et al. 2014, ApJ, 791, 81 Arnaud, K. A. 1996, Astronomical Data Analysis Software anc Systems V, 101, 17

Bigiel, F., Leroy, A., Walter, F., et al. 2008, AJ, 136, 2846

Bae, H.-J., \& Woo, J.-H. 2016, ApJ, 828, 97

Bae, H.-J., Woo, J.-H., Karouzos, M., et al. 2017, ApJ, 837, 9]

Blake, G. A., Sutton, E. C., Masson, C. R., \& Phillips, T. G 1987, ApJ, 315, 621

Canizares, C. R., Davis, J. E., Dewey, D., et al. 2005, PASP 117,1144

Cano-Díaz, M., Maiolino, R., Marconi, A., et al. 2012, A\&A, $537, \mathrm{~L} 8$

Cicone, C., Maiolino, R., Sturm, E., et al. 2014, A\&A, 562, A21

Cresci, G., Mainieri, V., Brusa, M., et al. 2015, ApJ, 799, 82

Cresci, G., Marconi, A., Zibetti, S., et al. 2015, A\&A, 582, A63

Croton, D. J., Springel, V., White, S. D. M., et al. 2006, MNRAS, 365, 11

Curran, S. J., Johansson, L. E. B., Rydbeck, G., \& Booth, R. S. 1998, A\&A, 338, 863

v http://cxc.harvard.edu/proposer/POG/html/chap8.html
Table A. Chandra Data for Supplemental Imaging Analysis

\begin{tabular}{ccccc}
\hline \hline ObsID & Obs. date (UT) & Grating & $\begin{array}{c}\text { Exp. } \\
(\mathrm{ksec})\end{array}$ & Tar. \\
$(1)$ & $(2)$ & $(3)$ & $(4)$ & $(5)$ \\
\hline 4771 & $2004 / 11 / 28$ & YES & 52 & $\mathrm{C}$ \\
10223 & $2008 / 12 / 15$ & YES & 88 & $\mathrm{~S}$ \\
10844 & $2008 / 12 / 24$ & YES & 22 & $\mathrm{~S}$ \\
10873 & $2009 / 03 / 01$ & YES & 16 & $\mathrm{~S}$ \\
10850 & $2009 / 03 / 03$ & YES & 12 & $\mathrm{~S}$ \\
10872 & $2009 / 03 / 04$ & YES & 14 & $\mathrm{~S}$ \\
10937 & $2009 / 12 / 28$ & NO & 10 & $\mathrm{~S}$ \\
$12823^{\dagger}$ & $2010 / 12 / 17$ & NO & 147 & $\mathrm{C}$ \\
$12824^{\dagger}$ & $2010 / 12 / 24$ & NO & 38 & $\mathrm{C}$ \\
& & Total & $399)$ & \\
\hline
\end{tabular}

\section{Notes.}

(1) Observation ID. (2) Observation start date. (3) Check on the grating observation. (4) Exposure after data reduction. (5) Target of each observation $(\mathrm{C}=$ Circinus and $\mathrm{S}=$ SN 1996cr). ${ }^{\dagger}$ These have been used in the main imaging analysis.

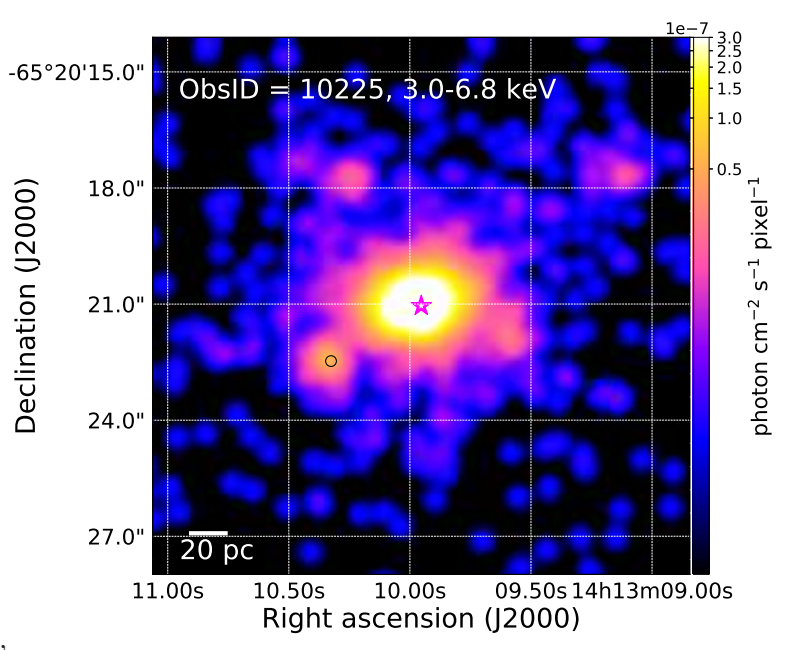

Fig. A. Broadband 3.0-6.8 keV image constructed from the ObsID = 10225 data. This demonstrates the emergence of a point source (black circle) around the CNR-SE region. 


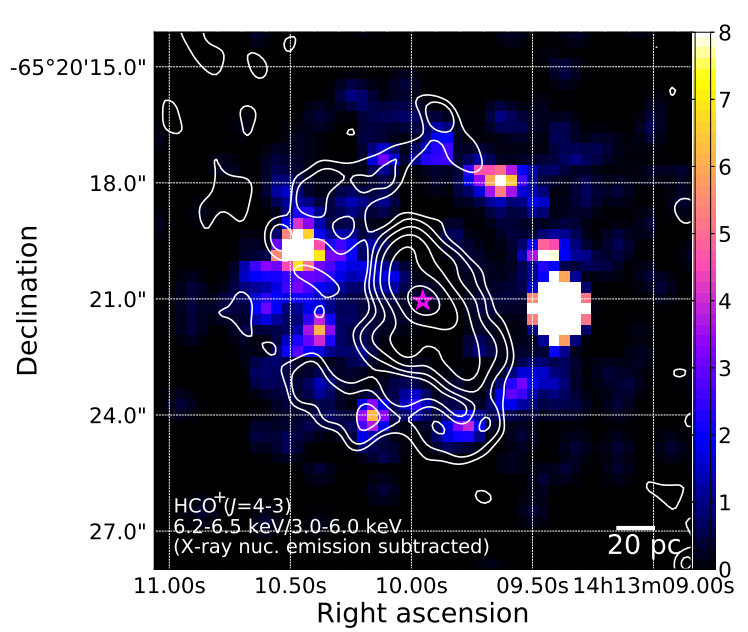

Fig. B. Same as Figure 7, but the X-ray image ratio is created by combining the data in Table $A$.

Curran, S. J., Rydbeck, G., Johansson, L. E. B., \& Booth, R. S. 1999, A\&A, 344, 767

Davis, J. E., Bautz, M. W., Dewey, D., et al. 2012, Proc. SPIE, $8443,84431 \mathrm{~A}$

Di Matteo, T., Springel, V., \& Hernquist, L. 2005, Nature, 433, 604

Elmouttie, M., Koribalski, B., Gordon, S., et al. 1998, MNRAS, 297,49

Elmouttie, M., Haynes, R. F., Jones, K. L., Sadler, E. M., \& Ehle, M. 1998, MNRAS, 297, 1202

Elmouttie, M., Krause, M., Haynes, R. F., \& Jones, K. L. 1998 , MNRAS, 300, 1119

Esquej, P., Alonso-Herrero, A., González-Martín, O., et al. 2014, ApJ, 780, 86

Fabbiano, G., Elvis, M., Paggi, A., et al. 2017, ApJL, 842, L4

Fabian, A. C. 2012, ARA\&A, 50, 455

Feruglio, C., Maiolino, R., Piconcelli, E., et al. 2010, A\&A, 518, L155

Feruglio, C., Fiore, F., Carniani, S., et al. 2015, A\&A, 583, A99

Fiore, F., Feruglio, C., Shankar, F., et al. 2017, A\&A, 601, A143

Freeman, K. C., Karlsson, B., Lynga, G., et al. 1977, A\&A, 55, 445

García, J., Dauser, T., Reynolds, C. S., et al. 2013, ApJ, 768, 146

García-Burillo, S., Combes, F., Usero, A., et al. 2014, A\&A, 567, A 125

Garmire, G. P., Bautz, M. W., Ford, P. G., Nousek, J. A., \& Ricker, G. R., Jr. 2003, Proc. SPIE, 4851, 28

Greve, T. R., Papadopoulos, P. P., Gao, Y., \& Radford, S. J. E. 2009, ApJ, 692, 1432

Greenhill, L. J., Booth, R. S., Ellingsen, S. P., et al. 2003, ApJ, 590,162

Harrison, C. M., Alexander, D. M., Mullaney, J. R., et al. 2012, ApJL, 760, L15
Harrison, C. M., Alexander, D. M., Mullaney, J. R., \& Swinbank, A. M. 2014, MNRAS, 441, 3306

Imanishi, M., Nakanishi, K., Yamada, M., Tamura, Y., \& Kohno, K. 2010, PASJ, 62, 201

Imanishi, M., \& Nakanishi, K. 2014, AJ, 148, 9

Imanishi, M., Nakanishi, K., \& Izumi, T. 2018, ApJ, 856, 143

Izumi, T., Kohno, K., Martín, S., et al. 2013, PASJ, 65, 100

Izumi, T., Kohno, K., Aalto, S., et al. 2016, ApJ, 818, 42

Izumi, T., Wada, K., Fukushige, R., Hamamura, S., \& Kohno, K. 2018, ApJ, 867, 48

Karachentsev, I. D., Makarov, D. I., \& Kaisina, E. I. 2013, AJ, 145, 101

Kawamuro, T., Ueda, Y., Tazaki, F., Ricci, C., \& Terashima, Y. 2016, ApJS, 225, 14

Kawamuro, T., Schirmer, M., Turner, J. E. H., Davies, R. L., \& Ichikawa, K. 2017, ApJ, 848, 42

Kennicutt, R. C., Jr., Calzetti, D., Walter, F., et al. 2007, ApJ, 671,333

Kohno, K. 2005, The Evolution of Starbursts, 783, 203

Koribalski, B. S., Staveley-Smith, L., Kilborn, V. A., et al. 2004, AJ, 128, 16

Krips, M., Neri, R., García-Burillo, S., et al. 2008, ApJ, 677, 262

LaMassa, S. M., Heckman, T. M., \& Ptak, A. 2012, ApJ, 758, 82

Lepp, S., \& Dalgarno, A. 1996, A\&A, 306, L21

Li, J., Kastner, J. H., Prigozhin, G. Y., \& Schulz, N. S. 2003 , ApJ, 590, 586

Li, J., Kastner, J. H., Prigozhin, G. Y., et al. 2004, ApJ, 610, 1204

Liu, G., Zakamska, N. L., Greene, J. E., Nesvadba, N. P. H., \& Liu, X. 2013, MNRAS, 436, 2576

Liu, G., Zakamska, N. L., Greene, J. E., Nesvadba, N. P. H., \& Liu, X. 2013, MNRAS, 430, 2327

Magdziarz, P., \& Zdziarski, A. A. 1995, MNRAS, 273, 837

Maiolino, R., Krabbe, A., Thatte, N., \& Genzel, R. 1998, ApJ, 493, 650

Maiolino, R., Alonso-Herrero, A., Anders, S., et al. 2000, ApJ, 531,219

Maiolino, R., Russell, H. R., Fabian, A. C., et al. 2017, Nature, 544,202

Maloney, P. R., Hollenbach, D. J., \& Tielens, A. G. G. M. 1996, ApJ, 466, 561

Maloney, P. R. 1999, Ap\&SS, 266, 207

Marconi, A., Moorwood, A. F. M., Origlia, L., \& Oliva, E. 1994, The Messenger, 78, 20

Marinucci, A., Risaliti, G., Wang, J., et al. 2012, MNRAS, 423, L6

Marinucci, A., Miniutti, G., Bianchi, S., Matt, G., \& Risaliti, G. 2013, MNRAS, 436, 2500

Marinucci, A., Bianchi, S., Fabbiano, G., et al. 2017, MNRAS, 470,4039

Matt, G., Fiore, F., Perola, G. C., et al. 1996, MNRAS, 281, L69

Matt, G., Guainazzi, M., Maiolino, R., et al. 1999, A\&A, 341, L39

Meijerink, R., \& Spaans, M. 2005, A\&A, 436, 397 
Meijerink, R., Spaans, M., \& Israel, F. P. 2007, A\&A, 461, 793

Mingo, B., Hardcastle, M. J., Croston, J. H., et al. 2012, ApJ, 758, 95

Morrison, R., \& McCammon, D. 1983, ApJ, 270, 119

McMullin, J. P., Waters, B., Schiebel, D., Young, W., \& Golap, K. 2007, Astronomical Data Analysis Software and Systems XVI, 376, 127

Moorwood, A. F. M., Lutz, D., Oliva, E., et al. 1996, A\&A, 315, L109

Mori, K., Tsunemi, H., Miyata, E., et al. 2001, New Century of X-ray Astronomy, 251, 576

Nandra, K., O'Neill, P. M., George, I. M., \& Reeves, J. N. 2007, MNRAS, 382, 194

Nobukawa, M., Koyama, K., Tsuru, T. G., Ryu, S. G., \& Tatischeff, V. 2010, PASJ, 62, 423

Oliva, E., Salvati, M., Moorwood, A. F. M., \& Marconi, A. 1994, A\&A, 288, 457

Oliva, E., Marconi, A., Cimatti, A., \& di Serego Alighieri, S. 1998, A\&A, 329, L21

Oosterloo, T., Raymond Oonk, J. B., Morganti, R., et al. 2017, A\&A, 608, A38

Page, M. J., Symeonidis, M., Vieira, J. D., et al. 2012, Nature, 485, 213

Prieto, M. A., Marco, O., \& Gallimore, J. 2005, MNRAS, 364, L28

Proga, D., Jiang, Y.-F., Davis, S. W., Stone, J. M., \& Smith, D. 2014, ApJ, 780, 51

Ricci, C., Ueda, Y., Koss, M. J., et al. 2015, ApJL, 815, L13

Robitaille, T., \& Bressert, E. 2012, Astrophysics Source Code Library, ascl:1208.017

Roche, P. F., Packham, C., Telesco, C. M., et al. 2006, MNRAS, 367,1689

Sambruna, R. M., Netzer, H., Kaspi, S., et al. 2001, ApJL, 546, L13

Silk, J., \& Rees, M. J. 1998, A\&A, 331, L1

Schöier, F. L., van der Tak, F. F. S., van Dishoeck, E. F., \& Black, J. H. 2005, A\&A, 432, 369

Smith, D. A., \& Wilson, A. S. 2001, ApJ, 557, 180

Soldi, S., Beckmann, V., Bassani, L., et al. 2005, A\&A, 444, 431

Sturm, E., González-Alfonso, E., Veilleux, S., et al. 2011, ApJL, 733, L16

Tanimoto, A., Ueda, Y., Kawamuro, T., et al. 2018, ApJ, 853, 146

Telesco, C. M., Pina, R. K., Hanna, K. T., et al. 1998, Proc. SPIE, 3354, 534

Terashima, Y., \& Wilson, A. S. 2001, ApJ, 560, 139

Tombesi, F., Meléndez, M., Veilleux, S., et al. 2015, Nature, 519,436

Tombesi, F., Veilleux, S., Meléndez, M., et al. 2017, ApJ, 850, 151

Tsunemi, H., Mori, K., Miyata, E., et al. 2001, ApJ, 554, 496

Usero, A., García-Burillo, S., Fuente, A., Martín-Pintado, J., \& Rodríguez-Fernández, N. J. 2004, A\&A, 419, 897

van der Tak, F. F. S., Black, J. H., Schöier, F. L., Jansen, D. J., \& van Dishoeck, E. F. 2007, A\&A, 468, 627

Viti, S., García-Burillo, S., Fuente, A., et al. 2014, A\&A, 570, A28
Wada, K. 2012, ApJ, 758, 66

Wada, K., Fukushige, R., Izumi, T., \& Tomisaka, K. 2018, ApJ, 852, 88

Wilson, A. S., Shopbell, P. L., Simpson, C., et al. 2000, AJ, 120, 1325

Woo, J.-H., Son, D., \& Bae, H.-J. 2017, ApJ, 839, 120

Yamada, M., Wada, K., \& Tomisaka, K. 2007, ApJ, 671, 73

Yang, Y., Wilson, A. S., Matt, G., Terashima, Y., \& Greenhill, L. J. 2009, ApJ, 691, 131

Young, A. J., Wilson, A. S., \& Shopbell, P. L. 2001, ApJ, 556, 6 